\title{
Article \\ Biofilm early stages growth and accumulation theoretical model
}

\author{
José M. Carvalho a,b , Manuel J. T. Carrondo a,c, and Luis L. Bonillab,e \\ a, LAQV-REQUIMTE, Chemistry Department, NOVA School of Science and Technology, Quinta da Torre, 2829-516 Caparica, \\ Portugal \\ b, Escuela Politécnica Superior, Universidad Carlos III de Madrid, Butarque 15, 28911 Leganés, Spain \\ c, Instituto de Biologia Experimental e Tecnológica (IBET), Apartado 12, 2781 - 901 Oeiras, Portugal \\ d, Instituto de Tecnologia Química e Biológica (ITQB), Universidade Nova de Lisboa, Apartado 127, 2781 - 901 Oeiras, Portugal \\ e, Instituto Gregorio Millan, Escuela Politecnica Superior, Universidad Carlos III de Madrid, Avda. de la Universidad 30, Leganes 28911, \\ Spain \\ * Correspondence: jmprc@fct.unl.pt
}

\begin{abstract}
A theoretical model to translate the evolution over time, in early stages, of growth and accumulation of biofilm bacterial mass is introduced. The model implies the solution of a system of differential-difference master equations.

The application of an algorithm like Miller's tree term recurrence, already known for Bessel functions of first kind, allows an exact calculation of the solutions of such equations, for a wide range of parameters values and time.

For biofilm model a five term recurrence is deduced and applied in a backwards computation. A suitable normalisation condition completes the reach of the solution.

Keywords: biofilm, Miller recurrent algorithm, Bessel functions, differential-difference master equations.
\end{abstract}

\section{Introduction}

Discrete mathematical models in space, and continuous in time, arise in many different contexts of Physics, Chemistry and Engineering.

Ever since, consideration of more sophisticated exact mathematical solutions, for transient regimes, invariantly leads, in known bibliography, to numerical and approximated methods application.

Such approach neglects all the theoretical information contained in an exact solution, which usually consists of special functions or, more frequently, complex combinations between their (in particular an infinite sum of terms with Bessel functions of first kind, modified or not).

As a matter of fact the accurate calculation of special functions seems far from being an inviting task for the vast majority of leading authors in several fields of exact sciences, namely Operational Research [1,2], Teletraffic Theory [3] and Manufacturing Systems [4].

Particularly notorious is the commentary in [2] and subsequent ones, with the same inspiration, many years after [5], and even nearly nowadays [6].

Functions like that one of transient $\mathrm{M} / \mathrm{M} / 1$ queue also came into play in Physical-Chemistry (nucleation, polymerization, crystallization, ... , aggregation processes in general), in Radar Theory [7], in Computer Networks Theory [8], and Congestion phenomenon [9]. 
From those exact solutions little information is brought to light, with the exception of long time behaviour (steady state) which is a limit easy to deduce. Normally transient scenery is avoided and quickly authors came on analysing steady state.

If transient regime is considered, having already an exact solution, a formalism of type Fokker-Planck is usually chosen to approximate such regime. The purpose is to mimic a process discrete in space and continuous in time by a process continuous both in space and time.

Doing things like that the initial infinite system of differential-difference equations is transformed in an equation with partial derivatives (Fokker-Planck equation).

On the other hand special functions, that are part of the solution or, even better saying, that are the "backbone" of the solution, are simply eliminated from all the analysis, and so we lose all their formal beauty.

Formalism conducting to Fokker-Planck equation started when Frenkel brought Becker-Doring theoretical work on nucleation kinetics to "its modern form", as Goodrich sais [10].

Seems that such "modern form", or others similar approximation approaches, has been generally accepted by the vast majority of authors in many domains of scientific knowledge already cited above.

In past recent years a theoretical model to account quantitatively evolution over time of growth and accumulation of biofilm biomass lead to a few similar exact solutions [11, chs. IV and VI].

In particular, the simplest version of all variants analysed them, lead to an analytical solution more complex than that of the $\mathrm{M} / \mathrm{M} / 1$ queue [11, pp. 67 and 70].

To compute the solution obtained with accuracy one must before deduce adequate homogeneous recurrence relations of a part of it containing, at least, an infinite sum of that solution already refereed. Even better if it is possible to deduce the homogeneous recurrence for the complete solution.

This has been in fact the case for the aforesaid simplest version of the biofilm model.

A suitable adaptation of the already known three terms recurrence (TTR) Miller algorithm [12] for the functions $J_{n}(x)$ and $I_{n}(x)$ to this new context in which recurrences have now more than tree terms has been done.

Miller applied backward recurrence "for evaluating sequences of functions $\left\{f_{k}\right\}$ when the recurrence connecting successive members was unstable for increasing $\mathrm{k}$ " as published Thacher [13].

Applying an algorithm like Miller's (TTR) [12], allowed to reach an exact calculation and graphic representation of such kind of solutions, with a five term recurrence, also applied in backwards calculation.

Consequently we are applying, in the simplest version of the biofilm model, a generalization of that algorithm including the whole infinite sum in functions $\left\{f_{k}\right\}$ and not only one Bessel function. So the recurrences obtained have more than tree terms, as expected according to the additional complexity of the functions.

The early stages of growth and accumulation of biofilm included in that model, was the subject of [11] jointly with the search of how to deal when facing theoretical models near the border of mathematic intractability.

Accordingly, and going deeper in that hard mathematical context, those early stages are considered more complicated than the referred simplest solution, which is only a first approach. 
Besides that first approach, using what we call "Mono-layered concentrated growth" kinetics, we, also for sake of completeness, described how to reach solutions for three more approaches named, Zero order kinetics, "Blackman inspired" kinetics and "Averaged" first order kinetics, all known in biotechnological literature.

Those approaches are, however, only particular cases of the main task, which is the mathematical deduction of the exact solution for Monod kinetic law.

We have already reach such general version of the model profiting the skills previously acquired solving the simplest version [11, ch. VI].

As consequence a theoretical Bessel functions framework seems to be an appropriate mathematical ambient for modeling biofilm formation and growth dynamics.

The ideas and the model described here are only the beginning of the modeling possibilities offered by that mathematic framework.

In Queuing Theory utilization of State-Transition-Rate Diagrams is a customary tool for description of a particular queue dynamics under analysis.

We also constructed the equivalent diagram for biofilm model, lightning this way an "isomorphism" between the two contexts [11, p. 182].

Accordingly with this circumstance, a careful analysis of Clarke classical solution [14] and our previous work [11] allows to put in equation and reach the transient solution of many more queues [11, ch. VII, pp. 167-180].

Such unifying way will allow, with a unique deduction, an exactly solved general solution.

As we can conclude, by the aforesaid considerations, transient phases in Queuing Theory is a subject, although not the only one, of most interest for our purposes. But we will leave that broader task for future works.

\section{Skope and structure of this work}

The theoretical model we introduce can be found in detail in [11, chs. I, II, III].

Here we only describe, in Part I, the mathematical resolution of the system of differential-difference master equations, and in Part II, an algorithm similar to Miller's tree term recurrence, already known for Bessel functions of first kind.

\section{Part I}

\section{Theoretical model resolution}

\section{Governing equation system definition}

We start the resolution of our model writing the total system of differential-difference master equations obtained in [11, ch. III, Box 5].

The system reads, from (1) to (4):

$$
\begin{aligned}
& \left(\frac{d S_{0}}{d t}\right)=-\boldsymbol{F} \cdot S_{0}+\left(k_{1}-\mu_{\text {máx. }}^{\vec{\prime}} \cdot \eta_{1}\right) \cdot S_{1}-\mu_{\text {máx. }} \cdot\left(\sum_{j=2}^{L} \eta_{j} \cdot S_{j}\right) \\
& \left(\frac{d S_{1}}{d t}\right)=\boldsymbol{F} \cdot S_{0}+\left[\left(\overrightarrow{\mu_{\text {máx. }}}-\mu_{\text {máx. }}^{\uparrow}\right) \cdot \eta_{1}-\boldsymbol{F}-k_{1}\right] \cdot S_{1}+\left[k_{2}+\mu_{\text {máx. }} \cdot\left(\eta_{2}-\eta_{1}\right)\right] \cdot S_{2}+
\end{aligned}
$$




$$
\begin{aligned}
& +\mu_{m a ́ x .} \cdot\left[\sum_{j=3}^{L}\left(\eta_{j}-\eta_{j-1}\right) \cdot S_{j}\right]-\overrightarrow{\mu_{m a ́ x}} \cdot \eta_{L} \cdot S_{L+1} \\
& \left(\frac{d S_{n}}{d t}\right)=\left[\boldsymbol{F}+\mu_{\text {máx. }}^{\uparrow} \cdot\left(\sum_{j=1}^{n-1} \eta_{j}\right)\right] \cdot S_{n-1}+\left[-\boldsymbol{F}+\mu_{\text {máx. }} \cdot \eta_{1}-\mu_{\text {máx. }}^{\uparrow} \cdot\left(\sum_{j=1}^{n} \eta_{j}\right)-k_{n}\right] \cdot S_{n}+ \\
& +\left[\overrightarrow{\mu_{\text {máx. }}} \cdot\left(\eta_{2}-\eta_{1}\right)+k_{n+1}\right] \cdot S_{n+1}+\mu_{\text {máx. }} \cdot\left[\sum_{j=2}^{L-1}\left(\eta_{j+1-} \eta_{j}\right) \cdot S_{n+j}\right]-\vec{\mu}_{\text {máx. }} \cdot \eta_{L} \cdot S_{n+L} \\
& \ldots \text { if }(2 \leq n \leq L) \\
& \left(\frac{d S_{n}}{d t}\right)=\left[\boldsymbol{F}+\mu_{\text {máx }}^{\uparrow} \cdot\left(\sum_{j=1}^{L} \eta_{j}\right)\right] \cdot S_{n-1}+\left[-\boldsymbol{F}+\mu_{\text {máx. }} \cdot \eta_{1}-\mu_{m a ́ x .}^{\uparrow} \cdot\left(\sum_{j=1}^{L} \eta_{j}\right)-k_{n}\right] \cdot S_{n}+ \\
& +\left[\mu_{\text {máx. }} \cdot\left(\eta_{2}-\eta_{1}\right)+k_{n+1}\right] \cdot S_{n+1}+\mu_{m a ́ x .} \cdot\left[\sum_{j=2}^{L-1}\left(\eta_{j+1-} \eta_{j}\right) \cdot S_{n+j}\right]-\mu_{m a ́ x .} \cdot \eta_{L} \cdot S_{n+L} \\
& \ldots \text { if }(n>L)
\end{aligned}
$$

And we recall the notation defined in [11, ch. II, pp. 11 to 19]

$S_{n}$ - area covered with n layers

$S_{T}$ - solid flat carrier total area

$L$ - maximum number of active layers in each area $S_{n}$. If $\mathrm{n}<\mathrm{L}$ all the layers are active, if $\mathrm{n}>\mathrm{L}$ only $\mathrm{L}$ layers are active, and $(\mathrm{n}-\mathrm{L})$ inactive.

$t$ - time

$\boldsymbol{F}$ - attachment flux of planktonic bacteria expressed in flat area covered over the total carrier area $S_{T}$

$k_{n}$ - kinetic constant for the process of biomass desorption (or detachment)

$\overrightarrow{\mu_{\text {máx. }}}$ - maximum specific growth rate in the direction parallel to the solid support

$\mu_{\text {máx. }}^{\uparrow}$ - maximum specific growth rate in the direction perpendicular to the solid support

$\eta_{j}$ - remaining factor, besides the maximums specific growth rates, in the Monod specific growth rate model. See [11, ch. III, pp. 25].

$j$-number, from 1 to $L$, and from top outermost layer to down innermost still active layer in any area $S_{n}$. See [11, ch. III, pp. 25].

This system accounts the variation with time of all $S_{n}(n=0,1,2, \ldots, n, \ldots)$.

All terms with common $S_{n}$ are colected together and are disposed in increased order $(n)$ in the right hand sides.

We observe that the system structure consists in,

$\left(\frac{d S_{0}}{d t}\right)$ : one differential equation

$\left(\frac{d S_{1}}{d t}\right)$ : one differential equation 
$\left(\frac{d S_{n}}{d t}\right)$ if $(2 \leq n \leq L):(L-1)$ differential equations

$\left(\frac{d S_{n}}{d t}\right)$ if $(n>L):$ infinite number of differential equations

The dimensionless variables and parameters that we choose are defined from (5) to (11):

$\tau=\left(\mu_{m a ́ x .}+\mu_{m a ́ x .}^{\uparrow}\right) \cdot \eta_{1} \cdot t$

$\theta_{n}=\frac{S_{n}}{S_{T}}$

$\rho=\frac{F}{\left(\mu_{\text {máx. }}^{\rightarrow}+\mu_{\text {máx. }}^{\uparrow}\right) \cdot \eta_{1}}$

$\Phi=\frac{\mu_{\text {máx. }}^{\uparrow}}{\left(\mu_{\text {máx. }}+\mu_{\text {máx. }}^{\uparrow}\right)}$

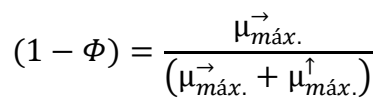

$\delta_{n}=\frac{k_{n}}{\left(\mu_{m \text { áx. }}+\mu_{m a ́ x .}^{\uparrow}\right) \cdot \eta_{1}}$

$\psi_{j}=\frac{\eta_{j}}{\eta_{1}}$

Before insert the dimensionless parameters it is convenient put in evidence the kinetic factor $\eta_{1}$ whenever it occurs to made easy the substitution of $\psi_{j}$. This is done from (12) to (15).

$$
\begin{aligned}
& \left(\frac{d S_{0}}{d t}\right)=-\boldsymbol{F} \cdot S_{0}+\left(k_{1}-\mu_{m a ́ x} \cdot \eta_{1}\right) \cdot S_{1}-\mu_{m a ́ x .} \cdot \eta_{1} \cdot\left[\sum_{j=2}^{L}\left(\frac{\eta_{j}}{\eta_{1}}\right) \cdot S_{j}\right]
\end{aligned}
$$

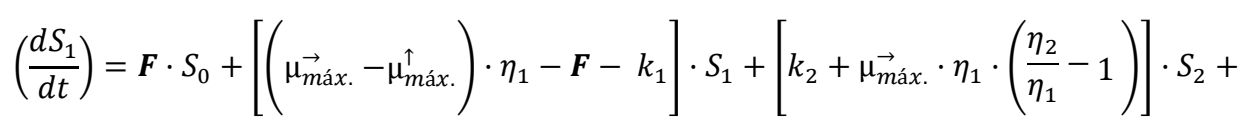

$$
\begin{aligned}
& +\mu_{m a ́ x .} \cdot \eta_{1} \cdot\left[\sum_{j=3}^{L}\left(\frac{\eta_{j}}{\eta_{1}}-\frac{\eta_{j-1}}{\eta_{1}}\right) \cdot S_{j}\right]-\mu_{m a ́ x} \cdot \eta_{1} \cdot\left(\frac{\eta_{L}}{\eta_{1}}\right) \cdot S_{L+1} \\
& \left(\frac{d S_{n}}{d t}\right)=\left\{\boldsymbol{F}+\mu_{\text {máx. }}^{\uparrow} \cdot \eta_{1} \cdot\left[\sum_{j=1}^{n-1}\left(\frac{\eta_{j}}{\eta_{1}}\right)\right]\right\} \cdot S_{n-1}-\left\{\boldsymbol{F}-\mu_{\text {máx. }} \cdot \eta_{1}+\mu_{\text {máx }}^{\uparrow} \cdot \eta_{1} \cdot\left[\sum_{j=1}^{n}\left(\frac{\eta_{j}}{\eta_{1}}\right)\right]+k_{n}\right\} \cdot S_{n}+ \\
& +\left[\overrightarrow{\mu_{\text {máx. }}} \cdot \eta_{1} \cdot\left(\frac{\eta_{2}}{\eta_{1}}-1\right)+k_{n+1}\right] \cdot S_{n+1}+\mu_{m a ́ x .} \cdot \eta_{1} \cdot\left[\sum_{j=2}^{L-1}\left(\frac{\eta_{j+1}}{\eta_{1}}-\frac{\eta_{j}}{\eta_{1}}\right) \cdot S_{n+j}\right]-\mu_{m a ́ x .} \cdot \eta_{1} \cdot\left(\frac{\eta_{L}}{\eta_{1}}\right) \cdot S_{n+L}
\end{aligned}
$$

$\ldots$ if $(2 \leq n \leq L)$

$$
\left(\frac{d S_{n}}{d t}\right)=\left\{\boldsymbol{F}+\mu_{\text {máx. }}^{\uparrow} \cdot \eta_{1} \cdot\left[\sum_{j=1}^{L}\left(\frac{\eta_{j}}{\eta_{1}}\right)\right]\right\} \cdot S_{n-1}-\left\{\boldsymbol{F}-\mu_{\text {máx. }} \cdot \eta_{1}+\mu_{m a ́ x}^{\uparrow} \cdot \eta_{1} \cdot\left[\sum_{j=1}^{L}\left(\frac{\eta_{j}}{\eta_{1}}\right)\right]+k_{n}\right\} \cdot S_{n}+
$$




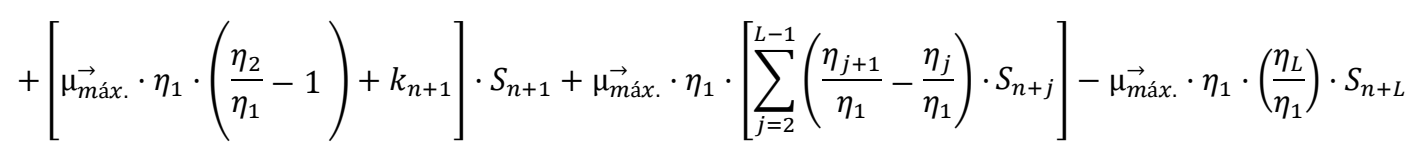

$\ldots$ if $(n>L)$

As consequence of (5) and (6) the derivatives $\left(\frac{d S_{n}}{d t}\right)$ on the left hand sides assume the form:

$$
\left(\frac{d S_{n}}{d t}\right)=\frac{d\left(\theta_{n} \cdot S_{T}\right)}{d\left[\frac{\tau}{\eta_{1}\left(\mu_{\text {máx. }}^{\overrightarrow{1}}+\mu_{\text {máx. }}^{\uparrow}\right)}\right]}=\left(\overrightarrow{\mu_{\text {máx. }}}+\mu_{\text {máx. }}^{\uparrow}\right) \cdot \eta_{1} \cdot S_{T} \cdot\left(\frac{d \theta_{n}}{d \tau}\right)
$$

The next step is to divide all the equations by $\left[\left(\mu_{m a ́ x}^{\vec{x}}+\mu_{\text {máx. }}^{\uparrow}\right) \cdot \eta_{1} \cdot S_{T}\right]$ and we get immediately the dimensionless system, equations (17) to (20). Besides, initial condition (21) is part of the system, consequently also must be included.

$$
\begin{gathered}
\left(\frac{d \theta_{0}}{d \tau}\right)=-\rho \cdot \theta_{0}+\left[\delta_{1}-(1-\Phi)\right] \cdot \theta_{1}-(1-\Phi) \cdot\left(\sum_{j=2}^{L} \psi_{j} \cdot \theta_{j}\right) \\
\left(\frac{d \theta_{1}}{d \tau}\right)=\rho \cdot \theta_{0}+\left[(1-2 \cdot \Phi)-\rho-\delta_{1}\right] \cdot \theta_{1}+\left[\delta_{2}+(1-\Phi) \cdot\left(\psi_{2}-1\right)\right] \cdot \theta_{2}+ \\
+(1-\Phi) \cdot\left[\sum_{j=3}^{L}\left(\psi_{j}-\psi_{j-1}\right) \cdot \theta_{j}\right]-(1-\Phi) \cdot \psi_{L} \cdot \theta_{L+1}
\end{gathered}
$$$$
\left(\frac{d \theta_{n}}{d \tau}\right)=\left[\rho+\Phi \cdot\left(\sum_{j=1}^{n-1} \psi_{j}\right)\right] \cdot \theta_{n-1}+\left[(1-\Phi)-\rho-\Phi \cdot\left(\sum_{j=1}^{n} \psi_{j}\right)-\delta_{n}\right] \cdot \theta_{n}+
$$$$
+\left[(1-\Phi) \cdot\left(\psi_{2}-1\right)+\delta_{n+1}\right] \cdot \theta_{n+1}+(1-\Phi) \cdot\left[\sum_{j=2}^{L-1}\left(\psi_{j+1}-\psi_{j}\right) \cdot \theta_{n+j}\right]-(1-\Phi) \cdot \psi_{L} \cdot \theta_{n+L}
$$

$$
\text { ... if }(2 \leq n \leq L)
$$

$$
\begin{aligned}
\left(\frac{d \theta_{n}}{d \tau}\right) & =\left[\rho+\Phi \cdot\left(\sum_{j=1}^{L} \psi_{j}\right)\right] \cdot \theta_{n-1}+\left[(1-\Phi)-\rho-\Phi \cdot\left(\sum_{j=1}^{L} \psi_{j}\right)-\delta_{n}\right] \cdot \theta_{n}+ \\
& +\left[(1-\Phi) \cdot\left(\psi_{2}-1\right)+\delta_{n+1}\right] \cdot \theta_{n+1}+(1-\Phi) \cdot\left[\sum_{j=2}^{L-1}\left(\psi_{j+1}-\psi_{j}\right) \cdot \theta_{n+j}\right]-(1-\Phi) \cdot \psi_{L} \cdot \theta_{n+L}
\end{aligned}
$$

The initial condition must, of course, be:

$$
\theta_{0}(0)=1 \text {, and } \theta_{n}(0)=0 \text { for } n>0
$$

(at the beginning $(\tau=0)$ al the solid support is bare) 


\section{General model resolution strategy: concept of "Mathematical meeting point"}

This system of equations is composed by the so-called differential equations of difference. It is a traditionally difficult domain and suitable theory for this subject can be found in Pinney [15], Bellman and Cooke [16] and El'sgol'ts and Norkin [17] and certainly other more recent works.

Also formally similar problems arise in Operational Research literature, in particular when transient states are analysed in Queuing Theory, both in book chapters and in articles.

In the present case the system has an analytical solution. However that solution can't be represented, for the most general version, by a closed form, like a final expression of type $\theta_{n}=\theta_{n}(\tau)$, and we will only solve the model for a simplified case and extract observations from that resolution.

Such observations will allow to establish the method for more general cases, in future work.

A formalism similar to that of Clarke ([14], [18]) will be our working tool. Suitable modifications will be introduced to adapt such solving way to the particular details of our case. Clarke formalism can also be found described in Saaty [19] and Srivastava and Kashyap [20].

We will use the following strategy, in two steps, being the first one the scope of this work.

The second one we will leaf for next future work:

First step: We must to solve the system for $L=1$ and $\delta_{1}=\delta_{2}=\cdots=\delta_{n}=\cdots=\delta$. That is, for the case where there is only one active layer and erosion is of equal intensity in all the surface fractions $\theta_{n}(n \geq 1)$. This one will be named the simplified version of the model. More specifically we can name this version the "Mono layered concentrated" growth kinetics variant, already defined in [11, ch. III].

Second step: profit from the solution obtained in the first step, inspecting their mathematical structure, and constructing a suitable Guess Solution that, as we will see in future work, will reduce all the solution of the complete system to an algebraic problem.

The algebraic problem defined in this second step falls in the context of algebraic difference equations and will be somewhat cumbersome. However it will be demonstrated that the complete resolution is possible.

The aforesaid Guess Solution contains an infinite series of Bessel functions, each one multiplied by a respective labelled factor. All those factors must then be analytically determined on a recurring suitable scheme.

Several mathematical and technical items, used in the first step, will be analyzed and selectively profited, with appropriate modifications, in the general resolution of the second step.

The system of the simplified "Mono layered concentrated" growth kinetics version, corresponding to the aforesaid first step, is easily obtained from (17) to (21) inserting the conditions:

... and,

$$
\delta_{1}=\delta_{2}=\cdots=\delta_{n}=\cdots=\delta
$$

$$
\psi_{j}=0, \ldots \ldots \ldots \text { for } j>1 \ldots \ldots \ldots \text { leaving } L \geq 2
$$

Condition (23) is equivalent to impose $L=1$ because all kinetic parameters $\psi_{j}$ are annulled for $j \geq 2$.

We also observe that (23) implicates the sameness between (18) and (19).

Consequently, the system for the simplified version is:

$\left(\frac{d \theta_{0}}{d \tau}\right)=-\rho \cdot \theta_{0}+[\delta-(1-\Phi)] \cdot \theta_{1}$

$\left(\frac{d \theta_{1}}{d \tau}\right)=\rho \cdot \theta_{0}+[(1-2 \cdot \Phi)-\rho-\delta] \cdot \theta_{1}+[\delta-(1-\Phi)] \cdot \theta_{2}$

$\left(\frac{d \theta_{n}}{d \tau}\right)=(\rho+\Phi) \cdot \theta_{n-1}+[(1-\Phi)-\rho-\Phi-\delta] \cdot \theta_{n}+[\delta-(1-\Phi)] \cdot \theta_{n+1}$

$$
\text { ... if }(n \geq 2)
$$

With initial condition:

$\theta_{0}(0)=1$, and $\theta_{n}(0)=0 \quad$ for $n>0$

We have already defined, as work context, [[11, p. 19, figs. 6 to 11] the five specific growth rate kinetics: 
- Monod kinetics

- Zero order kinetics

- "Blackman inspired" kinetics

- "Averaged" first order kinetics

- "Mono-layered concentrated growth" kinetics

When $L=1$ the first four (Monod, Zero order, "Blackman inspired" and "Averaged" first order) reduce in complexity and becomes equal to "Mono-layered concentrated growth" kinetics model. So we can conclude that to solve this last model is equivalent to solve the most simplified version of all the other four. We can consider "Mono-layered concentrated growth" kinetics as a sort of "mathematical meeting point" of all the kinetics under our analysis.

\section{5. "Mono layered concentrated" growth kinetics: exact solution}

\subsection{Common initial steps: generating function formalism}

The first step to start the resolution is to make the substitution,

$$
\theta_{n}(\tau)=e^{-(\rho+2 \cdot \Phi-\delta-1) \cdot \tau} \cdot Q_{n}(\tau)
$$

This substitution aims to simplify the system by eliminating the term relative to index $(n)$ on the right hand side, in all the equations for $(n \geq 1)$.

After simplification we get, from (29) to (31),

$\left(\frac{d Q_{0}}{d \tau}\right)=(2 \cdot \Phi+\delta-1) \cdot Q_{0}-(1-\Phi-\delta) \cdot Q_{1}$

$\left(\frac{d Q_{1}}{d \tau}\right)=\rho \cdot Q_{0}-(1-\Phi-\delta) \cdot Q_{2}$

$\left(\frac{d Q_{n}}{d \tau}\right)=(\rho+\Phi) \cdot Q_{n-1}-(1-\Phi-\delta) \cdot Q_{n+1}$

$$
\text { ... if }(n \geq 2)
$$

With initial condition:

$$
Q_{0}(0)=1, \quad \text { and } \quad Q_{n}(0)=0 \text { for } n>0
$$

Let now define the following generating function,

$$
G(z, \tau)=\sum_{n=0}^{\infty} a_{n} \cdot Q_{n}(\tau) \cdot \frac{(\tau-z)^{n}}{n !}
$$

... similar to that one of Clarke ([14], [18]),

$$
G(z, \tau)=\sum_{n=0}^{\infty} Q_{n}(\tau) \cdot \frac{(z-\tau)^{n}}{n !}
$$

... but with additional coefficients $a_{n}$ which remain to be determined and, for convenience of calculation, with changed signals at $\tau$ and $z$.

These modifications are justified because the system solved by Clarke is,

$\left(\frac{d Q_{0}}{d \tau}\right)=Q_{0}+Q_{1}$

$\left(\frac{d Q_{n}}{d \tau}\right)=\rho \cdot Q_{n-1}+Q_{n} \quad$... if $(n \geq 1)$

Therefore this is a simpler system because $\Phi$ and $\delta$ are null which is not the case of our system.

This explains the need to include the coefficients $a_{n}$.

Another difference lies in the exchanged signals in the functions $Q_{n}(\tau)$ which explains the need to change the signals in $\tau$ and $z$ in the generating function. 
Like in Clarke ([14], [18]) here we also seek for a generating function, $G(z, \tau)$ such that obeys a differential equation like,

$$
\frac{\partial^{2} G(z, \tau)}{\partial \tau \partial z}=\text { Constant } \cdot G(z, \tau)
$$

Which is the so called Telegraph equation.

The suitable deduction of coefficients $a_{n}$ will accomplish that goal.

First, $\frac{\partial^{2} G(z, \tau)}{\partial \tau \partial z}$ must be obtained from (33).

We get,

$$
\frac{\partial^{2} G(z, \tau)}{\partial \tau \partial z}=-a_{1} \cdot \frac{d Q_{1}}{d \tau}-\sum_{n=2}^{\infty} a_{n} \cdot \frac{d Q_{n}}{d \tau} \cdot \frac{(\tau-z)^{n-1}}{(n-1) !}-\sum_{n=2}^{\infty} a_{n} \cdot Q_{n} \cdot \frac{(\tau-z)^{n-2}}{(n-2) !}
$$

The order of partial derivations is indifferent,

$$
\frac{\partial^{2} G(z, \tau)}{\partial \tau \partial z}=\frac{\partial^{2} G(z, \tau)}{\partial z \partial \tau}
$$

Now, taking the expressions for $\frac{d Q_{n}}{d \tau}$ with $n \geq 1$ given by (30) and (31) we get,

$$
\begin{aligned}
\frac{\partial^{2} G(z, \tau)}{\partial \tau \partial z}=-a_{1} \cdot \rho \cdot Q_{0}-(\rho+\Phi) \cdot \sum_{n=2}^{\infty} a_{n} & \cdot Q_{n-1} \cdot \frac{(\tau-z)^{n-1}}{(n-1) !}- \\
& \quad-\sum_{n=2}^{\infty}\left[a_{n-1} \cdot(1-\Phi-\delta)-a_{n}\right] \cdot Q_{n} \cdot \frac{(\tau-z)^{n-2}}{(n-2) !}
\end{aligned}
$$

The usefulness of coefficients $a_{n}$ is now proved inspecting the second summation because we want to cancel it. The reason is that the exponents in $(\tau-z)$ is two units lower than the corresponding order of functions $Q_{n}$ and this summation cancelation would not be possible if all coefficients $a_{n}$ where equal to 1 , like in Clarke's generating function.

Determination of coefficients $a_{n}$ is easy and follows straightaway.

Must be,

$$
a_{n-1} \cdot(1-\Phi-\delta)-a_{n}=0 \quad \ldots \text { for }(n \geq 2)
$$

We get immediately the solution,

$$
a_{n}=a_{1} \cdot(1-\Phi-\delta)^{n-1} \quad \ldots \text { for }(n \geq 2)
$$

Substituting (42) in (40) the Telegraph equation now reads,

$$
\begin{aligned}
& \frac{\partial^{2} G(z, \tau)}{\partial \tau \partial z}= \\
& \quad=-(\rho+\Phi) \cdot(1-\Phi-\delta) \cdot\left[a_{1} \cdot \frac{\rho}{(\rho+\Phi) \cdot(1-\Phi-\delta)} \cdot Q_{0}+a_{1} \cdot \sum_{n=1}^{\infty}(1-\Phi-\delta)^{n-1} \cdot Q_{n} \cdot \frac{(\tau-z)^{n}}{(n) !}\right]
\end{aligned}
$$

Setting,

$$
a_{0}=a_{1} \cdot \frac{\rho}{(\rho+\Phi) \cdot(1-\Phi-\delta)}
$$

... equation (43) becomes,

$$
\frac{\partial^{2} G(z, \tau)}{\partial \tau \partial z}=-(\rho+\Phi) \cdot(1-\Phi-\delta) \cdot \sum_{n=0}^{\infty} a_{n} \cdot Q_{n} \cdot \frac{(\tau-z)^{n}}{(n) !}
$$

This right hand side is equal to $G(z, \tau)$ multiplied by $[-(\rho+\Phi) \cdot(1-\Phi-\delta)]$, which is constant. As proposed, a Telegraph equation is now reached. 
The coefficient $a_{0}$ can be equal to 1 for the sake of simplification. Consequently all the $a_{n}$ are now defined,

$$
\begin{gathered}
a_{0}=1 \\
\quad \ldots \text { and } \quad a_{n}=\frac{(\rho+\Phi)}{\rho} \cdot(1-\Phi-\delta)^{n} \quad \ldots \text { for }(n \geq 1)
\end{gathered}
$$

Till now holds,

$$
\frac{\partial^{2} G(z, \tau)}{\partial \tau \partial z}=-(\rho+\Phi) \cdot(1-\Phi-\delta) \cdot G(z, \tau) \quad \text { (Telegraph equation) }
$$

... where,

$$
G(z, \tau)=Q_{0}(\tau)+\frac{(\rho+\Phi)}{\rho} \cdot \sum_{n=1}^{\infty}(1-\Phi-\delta)^{n} \cdot Q_{n}(\tau) \cdot \frac{(z-\tau)^{n}}{n !}
$$

Now equation (29) and condition (32) will come into play.

According to (32), at time ( $\tau=0)$, the generating function must be $G(z, \tau)=Q_{0}(0)=1$.

So, condition (32) now reads. in generating function formalism,

$$
G(z, 0)=1
$$

With (49) the partial derivative $\left(\frac{\partial G}{\partial \tau}\right)$ can be easily obtained,

$$
\begin{aligned}
\left(\frac{\partial G}{\partial \tau}\right)=\frac{d Q_{0}}{d \tau}+\frac{(\rho+\Phi)}{\rho} \cdot \sum_{n=1}^{\infty}(1-\Phi-\delta)^{n} \cdot \frac{d Q_{n}}{d \tau} & \cdot \frac{(\tau-z)^{n}}{n !}+ \\
& +\frac{(\rho+\Phi)}{\rho} \cdot \sum_{n=1}^{\infty}(1-\Phi-\delta)^{n} \cdot Q_{n} \cdot \frac{(\tau-z)^{n-1}}{(n-1) !}
\end{aligned}
$$

When $(z=\tau)$,

$$
\left(\frac{\partial G}{\partial \tau}\right)_{z=\tau}=\frac{d Q_{0}}{d \tau}+\frac{(\rho+\Phi)}{\rho} \cdot(1-\Phi-\delta) \cdot Q_{1}
$$

Inserting here equation (29) one gets,

$$
\left(\frac{\partial G}{\partial \tau}\right)_{z=\tau}=(2 \cdot \Phi+\delta-1) \cdot Q_{0}+\frac{(1-\Phi-\delta) \cdot \Phi}{\rho} \cdot Q_{1}
$$

But,

$$
Q_{0}(\tau)=G(\tau, \tau)
$$

... and,

$$
\frac{\partial G}{\partial z}=-\frac{(\rho+\Phi)}{\rho} \cdot \sum_{n=1}^{\infty}(1-\Phi-\delta)^{n} \cdot Q_{n}(\tau) \cdot \frac{(\tau-z)^{n-1}}{(n-1) !}
$$

What means,

$$
\left(\frac{\partial G}{\partial z}\right)_{z=\tau}=-\frac{(\rho+\Phi)}{\rho} \cdot(1-\Phi-\delta) \cdot Q_{1}(\tau)
$$

... and, consequently,

$$
Q_{1}(\tau)=-\frac{\rho}{(\rho+\Phi) \cdot(1-\Phi-\delta)} \cdot\left(\frac{\partial G}{\partial z}\right)_{z=\tau}
$$

Equations (54) and (57) are now included in (53) and, in this way, the last condition for complete definition of the problem, in generating function context, is,

$$
\left(\frac{\partial G}{\partial \tau}\right)_{z=\tau}=(2 \cdot \Phi+\delta-1) \cdot G(\tau, \tau)-\frac{\Phi}{(\rho+\Phi)} \cdot\left(\frac{\partial G}{\partial z}\right)_{z=\tau}
$$

Once deduced, the generating function $G(z, \tau)$ will conduct easily to all the functions $Q_{n}(\tau)$. 
In fact from (49) one can conclude that,

$$
Q_{0}(\tau)=G(\tau, \tau) \quad \text { (already obtained before) }
$$

... and,

$$
Q_{n}(\tau)=-\frac{(-1)^{n} \cdot \rho}{(\rho+\Phi) \cdot(1-\Phi-\delta)^{n}} \cdot\left(\frac{\partial^{n} G}{\partial z^{n}}\right)_{z=\tau} \ldots \ldots \text { for }(n \geq 1)
$$

This last formula is easy to demonstrate, so we omit the details.

Lastly, with (28), fractions $\theta_{n}(\tau)$ are calculated from the functions $Q_{n}(\tau)$.

Then we will reach the solution of the simplified version of the model which is also the "mathematical meeting point" for all the kinetic variants under analysis.

A summary of what has been obtained so far in this generating function formalism, and will be used in the sequel, is, from (I) to (VII),

I) $\left(\frac{\partial^{2} G}{\partial z \partial \tau}\right)=-(\rho+\Phi) \cdot(1-\Phi-\delta) \cdot G(z, \tau)$

(Telegraph equation)

II) $G(z, 0)=1$

(Initial time condition)

III) $\left(\frac{\partial G}{\partial \tau}\right)_{z=\tau}=(2 \cdot \Phi+\delta-1) \cdot G(\tau, \tau)-$

$$
-\frac{\Phi}{(\rho+\Phi)} \cdot\left(\frac{\partial G}{\partial z}\right)_{z=\tau} \text { (First equation border condition) }
$$

IV) $\quad G(z, \tau)=Q_{0}(\tau)+\frac{(\rho+\Phi)}{\rho} \cdot \sum_{n=1}^{\infty}(1-\Phi-\delta)^{n} \cdot Q_{n}(\tau) \cdot \frac{(z-\tau)^{n}}{n !}$

(Generating function)

V) $\quad Q_{0}(\tau)=G(\tau, \tau)$

(Obtaining $Q_{0}$ )

VI) $\quad Q_{n}(\tau)=-\frac{(-1)^{n} \cdot \rho}{(\rho+\Phi) \cdot(1-\Phi-\delta)^{n}} \cdot\left(\frac{\partial^{n} G}{\partial z^{n}}\right)_{z=\tau} \ldots \ldots$ for $(n \geq 1)$

$$
\text { (Obtaining } Q_{n} \text { for } n \geq 1 \text { ) }
$$

VII) $\quad \theta_{n}(\tau)=e^{-(\rho+2 \cdot \Phi-\delta-1) \cdot \tau} \cdot Q_{n}(\tau)$

$$
\text { (Obtaining } \theta_{n} \text { from } Q_{n} \text { ) }
$$

Telegraph equation (48) is solved applying Laplace transform over variable $z$. Applying first to the left hand side, one gets,

$\mathcal{L}\left\{\frac{\partial}{\partial z} \cdot\left[\frac{\partial G}{\partial \tau}\right]\right\}=\int_{0}^{\infty} e^{-s \cdot z} \cdot \frac{\partial}{\partial z} \cdot\left[\frac{\partial G}{\partial \tau}\right] \cdot d z$ 
$\int_{0}^{\infty} e^{-s \cdot z} \cdot \frac{\partial}{\partial z} \cdot\left[\frac{\partial G}{\partial \tau}\right] \cdot d z=s \cdot \mathcal{L}\left[\frac{\partial G}{\partial \tau}\right]-\left(\frac{\partial G}{\partial \tau}\right)_{z=0}=s \cdot \frac{\partial g(s, \tau)}{\partial \tau}-f(\tau)$

The function $g(s, \tau)$ is the Laplace transform of $G(z, \tau)$ and,

$$
f(\tau)=\left[\frac{\partial G(z, \tau)}{\partial \tau}\right]_{z=0}
$$

Similarly, applying the transform to the right hand side,

$$
\mathcal{L}[-(\rho+\Phi) \cdot(1-\Phi-\delta) \cdot G(z, \tau)]=-(\rho+\Phi) \cdot(1-\Phi-\delta) \cdot g(s, \tau)
$$

The right had sides in (62) and (64) must be equal,

$$
s \cdot \frac{\partial g(s, \tau)}{\partial \tau}-f(\tau)=-(\rho+\Phi) \cdot(1-\Phi-\delta) \cdot g(s, \tau)
$$

The initial time condition (50) after Laplace transform reads,

$$
\mathcal{L}[G(z, 0)]=\mathcal{L}(1)
$$

... which is equivalent to,

$$
g(s, 0)=\frac{1}{s}
$$

The general solution of (65) is known from mathematical tables,

$$
g(s, \tau)=e^{-(\rho+\Phi) \cdot(1-\Phi-\delta) \cdot \frac{\tau}{s}} \cdot\left[\frac{1}{S} \cdot \int f(\tau) \cdot e^{-(\rho+\Phi) \cdot(1-\Phi-\delta) \cdot \frac{\tau}{s}} \cdot d \tau+C(s)\right]
$$

The integration "constant" $C(s)$ is only constant relatively to time $\tau$ and only depends on $s$. Inserting condition (67) and making $\tau=0$ one can get $C(s)$,

From (68),

$$
g(s, 0)=\frac{1}{s} \cdot\left[\int f(\tau) \cdot e^{(\rho+\Phi) \cdot(1-\Phi-\delta) \cdot \frac{\tau}{s}} \cdot d \tau\right]_{\tau=0}+C(s)
$$

Substitution of (67) here leads to,

$$
C(s)=\frac{1}{S} \cdot\left\{1-\left[\int f(\tau) \cdot e^{(\rho+\Phi) \cdot(1-\Phi-\delta) \cdot \frac{\tau}{s}} \cdot d \tau\right]_{\tau=0}\right\}
$$

Now is easy to particularize the general solution (68) for our case, inserting there this expression of $C(s)$,

$$
g(s, \tau)=\frac{e^{-(\rho+\Phi) \cdot(1-\Phi-\delta) \cdot \frac{\tau}{s}}}{s}+\int_{0}^{\tau} f(\sigma) \cdot \frac{e^{-(\rho+\Phi) \cdot(1-\Phi-\delta) \cdot \frac{(\tau-\sigma)}{S}}}{s} \cdot d \sigma
$$

An observation must be made about the change of $\tau$ by $\sigma$ as integration variable. This has been done with the purpose of avoid confusion between that variable and the superior limit in the integral.

\section{$\underline{5.2}$ Trifurcation stage: continuous biofilm, patchy biofilm and border case}


In the sequel, application of inverse Laplace transform leads back again to the generating function $G(z, \tau)$.

But before apply inverse transform tree hypotheses came into play depending on the relative value of parameter $\delta$ when compared to the value of $(1-\Phi)$. As bigger is $\delta$, or as littler is $(1-\Phi)$, more the process of erosion is important in their intensity, comparatively to the process of bacterial growth inside the biofilm.

In mathematical context this is compulsory because if the signal in the exponentials is positive the inverse transform is one, if it is null is another and if it is negative is yet another one.

Explicitly,

Case 1) If $\delta<(1-\Phi)$, the inverse transform reads,

$$
\mathcal{L}^{-1}\left\{\frac{e^{-a \cdot \frac{\tau}{s}}}{s}\right\}=J_{0}(2 \cdot \sqrt{a \cdot \tau \cdot z}) \ldots \ldots \text { where } a=(\rho+\Phi) \cdot(1-\Phi-\delta)>0
$$

Case 2) If $\delta>(1-\Phi)$, the inverse transform reads,

$$
\mathcal{L}^{-1}\left\{\frac{e^{+a \cdot \frac{\tau}{s}}}{s}\right\}=I_{0}(2 \cdot \sqrt{a \cdot \tau \cdot z}) \ldots \ldots \text { where } a=(\rho+\Phi) \cdot(\Phi+\delta-1)>0
$$

Case 3) If $\delta=(1-\Phi)$, the inverse transform reads,

$$
\mathcal{L}^{-1}\left\{\frac{1}{s}\right\}=1 \ldots \ldots \text { in this borderline case, } a=0
$$

The functions in cases 1 and 2 are respectively Bessel functions and Modified Bessel functions of first kind of order zero.

Bessel function of first kind of order $n$ is defined by,

$$
J_{n}(x)=\sum_{k=0}^{\infty} \frac{(-1)^{k}(x / 2)^{n+2 k}}{k ! \Gamma(n+k+1)}
$$

... and Modified Bessel function of first kind of order $n$ by,

$$
I_{n}(x)=\sum_{k=0}^{\infty} \frac{\left(\frac{x}{2}\right)^{n+2 k}}{k ! \Gamma(n+k+1)}
$$

Here $\Gamma(x)$ is the gamma function. When $x=1,2,3, \ldots$ then,

$$
\Gamma(x)=(x-1) !
$$

In our case $n=0$, so $\Gamma(n+k+1)=\Gamma(k+1)=k$ ! and we can write,

$$
J_{0}(x)=\sum_{k=0}^{\infty} \frac{(-1)^{k}(x / 2)^{2 k}}{(k !)^{2}}
$$

... and,

$$
I_{0}(x)=\sum_{k=0}^{\infty} \frac{(x / 2)^{2 k}}{(k !)^{2}}
$$

As indicated before, in the first stages of biofilm formation erosion is seldom the most intense process. In most of situations growth as consequence of active bacterial reproduction is nearly the only relevant process. This corresponds, in mathematical terms to the aforesaid case 1 . And we can classify the obtained biofilm as a "continuous biofilm". 
However, patchy biofilms, also exists elsewhere if erosion becomes strong enough in those initial times of development, leading to a steady state where bare areas of solid support till exist in finite extension, after a long time, and biomass remains only accumulated in colonies or clusters without a complete coverage of solid support area.

The designation "patchy biofilm" is opposed, or complementary to "continuous biofilm" in this physical classification.

Consequently we observe that this mathematical formalism can translate this duality in biofilm structure, relating different biofilm morphologies with different mathematical functions.

Such translation gets more visibility, principally when achieved formulas are graphically represented.

The border case, as will be demonstrated, corresponds to a steady state where the limit value of the bare fraction, $\theta_{0}(\tau)$, is zero at infinite time $\tau$, as expected.

Continuous biofilm, after some time, reach a stadium when coverage of initial support bare area is rendered complete. After that time $\tau$, the fraction $\theta_{0}(\tau)$ assume negative values, and those values are not physically possible. Consequently one must initiate a new calculation time interval where $\theta_{0}(\tau)$ no longer came into play and successively so on, later, with $\theta_{1}(\tau), \theta_{2}(\tau) \ldots$ by the same reasons. But this is beyond the scope of the present work.

The analytical solutions for the aforesaid tree cases are,

Solution for case 1: continuous biofilm: $[\delta<(1-\Phi)]$

$$
\theta_{n}(\tau)=\alpha_{n} \cdot e^{y} \cdot \sum_{k=0}^{\infty} \beta_{k} \cdot J_{n+k}(x)
$$

Where:

$$
\begin{aligned}
& x=2 \cdot \tau \cdot \sqrt{(\rho+\Phi) \cdot(1-\Phi-\delta)} \\
& y=(1-\rho-2 \cdot \Phi-\delta) \cdot \tau \\
& \alpha_{0}=1 \\
& \alpha_{n}=\frac{\rho \cdot(\rho+\Phi)^{n-1}}{\sqrt{(\rho+\Phi)^{n} \cdot(1-\Phi-\delta)^{n}}} \ldots \text { only for } n \geq 1 \\
& \beta_{0}=1 \\
& \beta_{1}=\frac{(2 \cdot \Phi+\delta-1)}{\sqrt{(\rho+\Phi) \cdot(1-\Phi-\delta)}} \\
& \beta_{k}=\frac{\left\{[\rho \cdot(1-\Phi-\delta)+\Phi \cdot(1-\delta)] \cdot \Phi^{k-1}+(\rho+1-\delta) \cdot(\Phi+\delta-1)^{k}\right\}}{(1-\delta) \cdot \sqrt{(\rho+\Phi)^{k} \cdot(1-\Phi-\delta)^{k}}} \quad \text {...only for } k \geq 2
\end{aligned}
$$

Solution for case 2: patchy biofilm: $[\delta>(1-\Phi)]$

$$
\theta_{n}(\tau)=\alpha_{n}^{*} \cdot e^{y} \cdot \sum_{k=0}^{\infty} \beta_{k}^{*} \cdot I_{n+k}\left(x^{*}\right)
$$

Where:

$$
\begin{aligned}
& x^{*}=2 \cdot \tau \cdot \sqrt{(\rho+\Phi) \cdot(\Phi+\delta-1)} \\
& y=(1-\rho-2 \cdot \Phi-\delta) \cdot \tau \\
& \alpha_{0}^{*}=1 \\
& \alpha_{n}^{*}=\frac{\rho \cdot(\rho+\Phi)^{n-1}}{\sqrt{(\rho+\Phi)^{n} \cdot(\Phi+\delta-1)^{n}}} \ldots \text { only for } n \geq 1
\end{aligned}
$$




$$
\begin{aligned}
& \beta_{0}^{*}=1 \\
& \beta_{1}^{*}=\frac{(2 \cdot \Phi+\delta-1)}{\sqrt{(\rho+\Phi) \cdot(\Phi+\delta-1)}} \\
& \beta_{k}^{*}=\frac{\left\{[\rho \cdot(1-\Phi-\delta)+\Phi \cdot(1-\delta)] \cdot \Phi^{k-1}+(\rho+1-\delta) \cdot(\Phi+\delta-1)^{k}\right\}}{(1-\delta) \cdot \sqrt{(\rho+\Phi)^{k} \cdot(\Phi+\delta-1)^{k}}} \quad \text {... only for } k \geq 2
\end{aligned}
$$

Solution for case 3: border case between cases 1 and 2: $[\delta=(1-\Phi)]$

$$
\begin{aligned}
& \theta_{0}(\tau)=e^{-\rho \tau} \\
& \theta_{n}(\tau)=e^{-(\rho+\Phi) \cdot \tau} \cdot \frac{\rho \cdot(\rho+\Phi)^{n-1}}{\Phi^{n}}\left\{e^{\Phi \tau}-\sum_{k=0}^{n-1} \frac{(\tau \cdot \Phi)^{k}}{k !}\right\} \ldots \text { for }(n>0)
\end{aligned}
$$

And we provide the demonstration of these tree solutions respectively in Appendix A, Appendix B and Appendix C.

\section{$\underline{5.3}$ Concluding remark in Part I.}

All the consistency and coherence demonstrated about the changes between the solutions of the three analysed cases, considering in each transformation the appropriate mathematics modifications, leaves to conclude that a unique recurrent algorithm is enough for applications. In fact, the solution of case 1 conglobates the other two, after suitable modifications.

\section{$\underline{\text { Part II }}$}

\section{Miller Generalized Algorithm (eroding mathematical intractability)}

\section{Preliminary introduction.}

The solution reached in Part I for the "Mono-layered concentrated growth" kinetics, defined by formulas (80) for continuous biofilm (case 1) and by (81) for patchy biofilm (case 2) are much more difficult to handle than the frontier between those two (case 3) given by (82(0)) and $(82(n))$. Besides the case 3 is the less important, representing only a very specific choice of parameters with the purpose of corroborate the mathematical coherence in all the deductions made. From these proof we concluded that to handle case 1 solution (80), leads to a mathematical difficulty similar to handle case 2 solution (81). And also leads to the same recurrent result.

Consequently we focus now our attention exclusively in continuous biofilm solution (80).

This solution is not easy to handle for numeric calculation and therefore also not easy for graphic and intelligible representation of the model.

The solution obtained is complex because it consists of an infinite series of terms and the main difficulty lies on the fact that each term, in the infinite sum, contains a Bessel function of first kind, $J_{n+k}(x)$.

It is not because the summation is infinite in the number of terms why it is difficult reach a rigorous calculation. The truth is that terms of bigger order will always diminish in absolute value, and this circumstance allows, sooner or later, to despise those upper order terms becoming possible to accomplish all the computation without error. 
The main problem to solve refers to the rigorous calculation of each Bessel function not only because extensive ranges of orders $(n+k)$ are required but also because their argument values, proportional with dimensionless time $(\tau)$, must vary over a large interval. As a matter of fact, we do not always find values for arguments that, in our case, are (or can be) relatively high. There is out of question have time scale limitations when analysing a transient exact solution.

A priori do not exist tables providing such exigent demands on Bessel function orders and argument values.

The infinite series of terms with Bessel functions, which we have obtained in our case, can't (or should not) be calculated using published tables, not only because it is necessary to use very high order functions, not always tabulated, but also because, even if the tables have these high order Bessel functions, the accuracy is not the same as the lower-order functions $\left(J_{0}(x), J_{1}(x), J_{2}(x), \ldots\right)$.

This is an additional inconvenient question related to want of accuracy uniformity in data provided by known tables. Bessel functions of lower order $(0,1,2, \ldots)$ usually are tabulated with much more decimal significant places than the others (Abramowitz and Stegun, [21]). Thus, when one intends to calculate some more terms in the series, in the purpose of not to neglect meaningful terms, coherence is lost because their accuracy is almost always inferior to that of the initial terms.

Consequently we must work only with computer tools paying attention to achieve the better exactitude disposable nowadays.

We choose to compute, in future work, in Excel spreadsheet not only because it stores numerical values as "Double Precision Floating Point", representing numbers accurate to something like 15 decimal places, but also because it allows to design the layout of the algorithms, over the spreadsheet with great personalized liberty.

Another important advantage lies on the fact that one can "see" the contents of each cell and easily detect and correct errors and (or) improve the global efficiency of the entire algorithm.

Once discharged the numeric values published in those tables our attention goes to the theoretic method used, in old past epochs, for construct such Bessel functions tables.

The central and fundamental tool for such purpose was the Three Terms Recurrence (TTR) Miller's algorithm (Miller, [22]).

The immediate obvious way to solve the problem, of handle solution (80) with adequate accuracy, is to apply TTR Miller's algorithm, term by term, till reach a Bessel function order high enough for neglect the absolute value of such term and also the absolute values of all following terms.

Another, more efficient and elegant, alternative concerns with considering all the infinite series summation, and so all the function $\theta_{n}(\tau)$, as a special function passible for application of an algorithm related to, but more general than, TTR Miller's algorithm.

In more explicit words: functions $\theta_{n}(\tau)$ will came into play relatively to this new algorithm in a similar way Bessel functions of first kind play their role relatively to the already known TTR Miller's algorithm.

Application of this new approach implies, as first step, the deduction of a corresponding homogeneous recurrence for the special functions $\theta_{n}(\tau)$.

In the following section we will deduce that homogeneous recurrence and explain how can it be used for accurate computation of functions $\theta_{n}(\tau)$, translating to this new method a similar reasoning to that one of the old TTR Miller's algorithm method. 
These two methods can be jointly, and independently, applied and so we will be able to validate mutually both, in next future work.

\section{Generalized Miller's like algorithm construction.}

The calculation of the model equation (80), will be done, in this section, applying a method alike that of Miller [22] but more general.

This method applies to the so-called Special Functions when one can construct recurrence formulas between them. Knowing the functions of lower orders we can calculate those of higher orders with a formula of progressive recurrence and vice versa with a regressive one.

However, if the Special Functions are decreasing when their order increases the application of the progressive recurrence will imply the use of the subtraction operation and the loss of significant numbers (S. Zhang and J. Jin, [23]) each time the recurrence computation applies, making the method numerically unstable.

This is the case for Bessel functions of first kind and therefore also the case for the functions $\theta_{n}(\tau)$ at the initial values of $(\tau)$. But not only in the initial values of $(\tau)$ since there will always exist surface fractions $\theta_{n}(\tau)$, in the largest orders $(n)$ that are in stage of increasing its value, having therefore a low value, since the kinetic process is an overall continuous accumulation of biomass.

Therefore, it is necessary to use a regressive formula in all values of dimensionless time $(\tau)$. That is the same to say that we have to compute the functions $\theta_{n}(\tau)$ with the smallest orders $(n)$ depending of those ones with the biggest orders $(n)$.

Miller [22] solved the problem by assigning an arbitrary value to the function from where the recurrence computation begins, which is the one of the highest $\operatorname{order}(n)$.

By applying the homogeneous recurrence formula one gets the relative correct magnitudes between all the functions under calculation. After a suitable common normalization factor must be applied to all the functions of the sequence. The result of this multiplication is the exact value of all and each one of the functions.

The aforesaid suitable common normalization factor is defined by the known value, in the particular case under analysis, of an infinite series with form,

$$
\sum_{n=0}^{\infty} c_{n} \cdot f_{n}(\tau)
$$

The homogeneous recurrence formula to be constructed must, for the functions $\theta_{n}(\tau)$, read like,

$$
\theta_{n}(\tau)=F\left[\theta_{n+1}(\tau), \theta_{n+2}(\tau), \ldots, \theta_{n+k}(\tau)\right]
$$

... where,

$$
k \geq 1
$$

... and being $\theta_{n}(\tau)$ obtained depending of $\theta_{n+1}(\tau), \theta_{n+2}(\tau), \ldots, \ldots$ and $\theta_{n+k}(\tau)$.

We call (84) a $(k+1)$-terms homogeneous recurrence.

Let's go now to the homogeneous recurrence deduction, and for start, let define the auxiliary functions $W_{n}(x)$, for $(n \geq 0)$,

$$
W_{n}(x)=\sum_{k=0}^{\infty} \beta_{k} \cdot J_{n+k}(x)
$$




$$
W_{n}(x)=\beta_{0} \cdot J_{n}(x)+\beta_{1} \cdot J_{n+1}(x)+\sum_{k=2}^{\infty} \beta_{k} \cdot J_{n+k}(x)
$$

... where coefficients $\beta_{k}$ are defined like they have been defined in the model equation (80),

$$
\beta_{k}=\left\{\begin{array}{l}
1 \ldots \text { if } k=0 \\
\frac{b_{k-1} \ldots \text { if } k \geq 1}{\gamma^{k}} \text {... being, } \gamma=\sqrt{(1-\Phi-\delta) \cdot(\rho+\Phi)} \ldots \text { and }, b_{k}=c_{k} \cdot k !
\end{array}\right.
$$

Following a brief revision from Appendix A, coefficients $c_{k}$ are, in their turn, defined by equations $(A 19(0))$ and $(A 29)$,

$$
c_{k}=\left\{\begin{array}{l}
(2 \cdot \Phi+\delta-1) \ldots \text { if } k=0 \\
\frac{\left\{[\rho \cdot(1-\Phi-\delta)+\Phi \cdot(1-\delta)] \cdot \Phi^{k}+(\rho+1-\delta) \cdot(\Phi+\delta-1)^{k+1}\right\}}{(1-\delta) \cdot k !} \ldots \text { if } k \geq 1
\end{array}\right.
$$

And, also from Appendix A, consequently the coefficients $b_{k}$ obey equation $(A 20)$,

$$
b_{k+2}+A \cdot b_{k+1}+B \cdot b_{k}=0 \quad \ldots \text { with }(k \geq 0)
$$

... where,

$$
\begin{aligned}
& A=(1-2 \cdot \Phi-\delta) \\
& B=\Phi \cdot(\Phi+\delta-1)
\end{aligned}
$$

Let use functions $W_{n}(x)$ in the form,

$$
W_{n}(x)=J_{n}(x)+\sum_{k=1}^{\infty} \frac{b_{k-1}}{\gamma^{k}} \cdot J_{n+k}(x)
$$

Our purpose is now to obtain a recurrence relation between the functions $W_{n}(x)$ in which the functions $J_{n}(x)$ are not present. And, in the sequel, applying the relationship between functions $W_{n}(x)$ and functions $\theta_{n}(\tau)$, that recurrence will be easily converted into a recurrence between the functions $\theta_{n}(\tau)$, as desired.

Recurrences concerning Bessel functions of first kind imply $J_{n}(x), J_{n+1}(x)$ and $J_{n+2}(x)$. For this reason let now write explicitly $W_{n}(x), W_{n+1}(x)$ and $W_{n+2}(x)$ in the following way,

$$
\begin{aligned}
& W_{n}(x)=J_{n}(x)+\frac{b_{0}}{\gamma} \cdot J_{n+1}(x)+\frac{b_{1}}{\gamma^{2}} \cdot J_{n+2}(x)+\sum_{k=3}^{\infty} \frac{b_{k-1}}{\gamma^{k}} \cdot J_{n+k}(x) \\
& W_{n+1}(x)=J_{n+1}(x)+\frac{b_{0}}{\gamma} \cdot J_{n+2}(x)+\sum_{k=2}^{\infty} \frac{b_{k-1}}{\gamma^{k}} \cdot J_{n+k+1}(x) \\
& W_{n+2}(x)=J_{n+2}(x)+\sum_{k=1}^{\infty} \frac{b_{k-1}}{\gamma^{k}} \cdot J_{n+k+2}(x)
\end{aligned}
$$

Remodelling the summations so that everyone starts with $(k=1)$,

$$
W_{n}(x)=J_{n}(x)+\frac{b_{0}}{\gamma} \cdot J_{n+1}(x)+\frac{b_{1}}{\gamma^{2}} \cdot J_{n+2}(x)+\sum_{k=1}^{\infty} \frac{b_{k+1}}{\gamma^{k+2}} \cdot J_{n+k+2}(x)
$$


$W_{n+1}(x)=J_{n+1}(x)+\frac{b_{0}}{\gamma} \cdot J_{n+2}(x)+\sum_{k=1}^{\infty} \frac{b_{k}}{\gamma^{k+1}} \cdot J_{n+k+2}(x)$

$W_{n+2}(x)=J_{n+2}(x)+\sum_{k=1}^{\infty} \frac{b_{k-1}}{\gamma^{k}} \cdot J_{n+k+2}(x)$

Now we properly multiply (89(1)) and (89(2)) in such a way that, by associating the same Bessel function in the three equations (89(0)), (89(1)) and (89(2)), equality (A20) can be used thus eliminating the infinite terms summation:

$W_{n}(x)=J_{n}(x)+\frac{b_{0}}{\gamma} \cdot J_{n+1}(x)+\frac{b_{1}}{\gamma^{2}} \cdot J_{n+2}(x)+\sum_{k=1}^{\infty} \frac{b_{k+1}}{\gamma^{k+2}} \cdot J_{n+k+2}(x)$

$\frac{A}{\gamma} \cdot W_{n+1}(x)=\frac{A}{\gamma} \cdot J_{n+1}(x)+\frac{A \cdot b_{0}}{\gamma^{2}} \cdot J_{n+2}(x)+\sum_{k=1}^{\infty} \frac{A \cdot b_{k}}{\gamma^{k+2}} \cdot J_{n+k+2}(x)$

$\frac{B}{\gamma^{2}} \cdot W_{n+2}(x)=\frac{B}{\gamma^{2}} \cdot J_{n+2}(x)+\sum_{k=1}^{\infty} \frac{B \cdot b_{k-1}}{\gamma^{k+2}} \cdot J_{n+k+2}(x)$

Adding the three equations (90(0)), (90(1)) and (90(2)) we obtain,

$$
\begin{aligned}
& W_{n}(x)+\frac{A}{\gamma} \cdot W_{n+1}(x)+\frac{B}{\gamma^{2}} \cdot W_{n+2}(x)=J_{n}(x)+\frac{\left(b_{0}+A\right)}{\gamma} \cdot J_{n+1}(x)+ \\
& +\frac{\left(b_{1}+A \cdot b_{0}+B\right)}{\gamma^{2}} \cdot J_{n+2}(x)+\sum_{k=1}^{\infty} \frac{\left(b_{k+1}+A \cdot b_{k}+B \cdot b_{k-1}\right)}{\gamma^{k+2}} \cdot J_{n+k+2}(x)
\end{aligned}
$$

Observing that, from $b_{k}=c_{k} \cdot k !$, and also $(A 19(0)),(A 19(1)),(A 22)$ and (A23), the following three equalities are valid,

$b_{0}+A=0$

(92)

$b_{1}+A \cdot b_{0}+B=\gamma^{2}$

(93)

$b_{k+1}+A \cdot b_{k}+B \cdot b_{k-1}=0 \quad \ldots$ for $(k \geq 1)$

... we are now able to insert they in (91), and therefore getting in it a great simplification,

$$
W_{n}(x)+\frac{A}{\gamma} \cdot W_{n+1}(x)+\frac{B}{\gamma^{2}} \cdot W_{n+2}(x)=J_{n}(x)+J_{n+2}(x)
$$

Let now add 2 in the discrete order $(n)$ at (95),

$$
W_{n+2}(x)+\frac{A}{\gamma} \cdot W_{n+3}(x)+\frac{B}{\gamma^{2}} \cdot W_{n+4}(x)=J_{n+2}(x)+J_{n+4}(x)
$$

... and call into play the identities,

$$
J_{n}(x)+J_{n+2}(x)=\frac{2 \cdot(n+1)}{x} \cdot J_{n+1}(x)
$$




$$
J_{n+2}(x)+J_{n+4}(x)=\frac{2 \cdot(n+3)}{x} \cdot J_{n+3} 147(x)
$$

... which must be substituted in (95) and (96),

$$
\begin{aligned}
& W_{n}(x)+\frac{A}{\gamma} \cdot W_{n+1}(x)+\frac{B}{\gamma^{2}} \cdot W_{n+2}(x)=\frac{2 \cdot(n+1)}{x} \cdot J_{n+1}(x) \\
& W_{n+2}(x)+\frac{A}{\gamma} \cdot W_{n+3}(x)+\frac{B}{\gamma^{2}} \cdot W_{n+4}(x)=\frac{2 \cdot(n+3)}{x} \cdot J_{n+3}(x)
\end{aligned}
$$

... reducing this way to only one the Bessel functions of first kind figured in the right hand sides.

Now we explicit those Bessel functions in the left hand side,

$$
\begin{aligned}
& J_{n+1}(x)=\frac{x}{2 \cdot(n+1)} \cdot\left\{W_{n}(x)+\frac{A}{\gamma} \cdot W_{n+1}(x)+\frac{B}{\gamma^{2}} \cdot W_{n+2}(x)\right\} \\
& J_{n+3}(x)=\frac{x}{2 \cdot(n+3)} \cdot\left\{W_{n+2}(x)+\frac{A}{\gamma} \cdot W_{n+3}(x)+\frac{B}{\gamma^{2}} \cdot W_{n+4}(x)\right\}
\end{aligned}
$$

Back to equation (95) let write it again but adding this time 1 in the discrete order $(n)$,

$$
W_{n+1}(x)+\frac{A}{\gamma} \cdot W_{n+2}(x)+\frac{B}{\gamma^{2}} \cdot W_{n+3}(x)=J_{n+1}(x)+J_{n+3}(x)
$$

The right hand side at (102) only depends on $J_{n+1}(x)$ and $J_{n+3}(x)$ which in turn are given by (100) and (101) depending only on $W_{n+k}(x)$ with $k=0,1,2,3$ or 4 .

Inserting the equations (100) and (101) in the right hand side at (102) we obtain an equation where all Bessel functions of first kind have been eliminated by suitable substitutions, as desired.

That result reads,

$$
\begin{aligned}
W_{n+1}(x)+\frac{A}{\gamma} \cdot W_{n+2}(x)+ & \frac{B}{\gamma^{2}} \cdot W_{n+3}(x) \\
=\frac{x}{2 \cdot(n+1)} \cdot & \left\{W_{n}(x)+\frac{A}{\gamma} \cdot W_{n+1}(x)+\frac{B}{\gamma^{2}} \cdot W_{n+2}(x)\right\}+ \\
& +\frac{x}{2 \cdot(n+3)} \cdot\left\{W_{n+2}(x)+\frac{A}{\gamma} \cdot W_{n+3}(x)+\frac{B}{\gamma^{2}} \cdot W_{n+4}(x)\right\}
\end{aligned}
$$

The expression we have reached, (103), can now be transformed into a progressive recurrence if we explicit $W_{n+4}(x)$ as depending of the functions of lesser orders, or in a regressive recurrence if we explicit $W_{n}(x)$ as depending of all the others which have higher orders.

For the sake to attain numerical stability we are interested in the regressive recurrence for a proper application of the Generalized Miller's like algorithm we seek for construction.

Before to manipulate (103) is convenient establish an alternative expression for $\left(\frac{2}{x}\right)$.

From the definitions $x=2 \cdot \tau \cdot \sqrt{(\rho+\Phi) \cdot(1-\Phi-\delta)}$ and $\gamma=\sqrt{(\rho+\Phi) \cdot(1-\Phi-\delta)}$ the result follows immediately,

$$
\frac{2}{x}=\frac{1}{\gamma \cdot \tau}
$$

The wanted regressive recurrence is now reached easily from (103), taking (104) in consideration,

$$
\begin{aligned}
W_{n}(x)=\left[\frac{(n+1)}{\gamma \cdot \tau}-\frac{A}{\gamma}\right] & \cdot W_{n+1}(x)+\left[\frac{(n+1) \cdot A}{\gamma^{2} \cdot \tau}-\frac{B}{\gamma^{2}}-\frac{(n+1)}{(n+3)}\right] \cdot W_{n+2}(x)+ \\
& +\left[\frac{(n+1) \cdot B}{\gamma^{3} \cdot \tau}-\frac{(n+1) \cdot A}{(n+3) \cdot \gamma}\right] \cdot W_{n+3}(x)-\frac{(n+1) \cdot B}{(n+3) \cdot \gamma^{2}} \cdot W_{n+4}(x)
\end{aligned}
$$


What logically follows must be to relate functions $W_{n}(x)$ with functions $\theta_{n}(\tau)$ and then insert that relation in (105) and finally obtain a regressive recurrence for these last one functions.

From the model solution, (80) and the definition of $W_{n}(x),(87)$,

$$
\begin{aligned}
& \theta_{0}(\tau)=e^{y} \cdot W_{0}(x) \\
\theta_{n}(\tau)= & \frac{\rho \cdot(\rho+\Phi)^{n-1}}{\gamma^{n}} \cdot e^{y} \cdot W_{n}(x) \quad \ldots \text { for }(n \geq 1)
\end{aligned}
$$

Next step is to obtain explicit formulas for $W_{n}(x)$ depending on $\theta_{n}(\tau)$,

$$
\begin{gathered}
W_{0}(x)=e^{-y} \cdot \theta_{0}(\tau) \\
W_{n}(x)=e^{-y} \cdot \frac{\gamma^{n}}{\rho \cdot(\rho+\Phi)^{n-1}} \cdot \theta_{n}(\tau) \ldots \text { for }(n \geq 1)
\end{gathered}
$$

Regarding the fact that the function $W_{0}(x)$ has a dependence, different from all the others, on the corresponding function $\theta_{0}(\tau)$ one is compelled to conclude that also a different and unique regressive recurrence relation must be established for $\theta_{0}(\tau)$.

Accordingly, inserting $(107(0))$ and $(107(n))$ in (105), and making $(n=0)$, the recurrence for $\theta_{0}(\tau)$ is achieved and reads,

$$
\begin{aligned}
\theta_{0}(\tau)=\left[\frac{1}{\tau}-A\right] \cdot \frac{\theta_{1}(\tau)}{\rho}+\left[\frac{A}{\tau}-\right. & \left.B-\frac{\gamma^{2}}{3}\right] \cdot \frac{\theta_{2}(\tau)}{\rho \cdot(\rho+\Phi)}+ \\
& +\left[\frac{B}{\tau}-\frac{A \cdot \gamma^{2}}{3}\right] \cdot \frac{\theta_{3}(\tau)}{\rho \cdot(\rho+\Phi)^{2}}-\frac{B \cdot \gamma^{2}}{3} \cdot \frac{\theta_{4}(\tau)}{\rho \cdot(\rho+\Phi)^{3}}
\end{aligned}
$$

... and inserting only $(107(n))$ in (105), and making $(n \geq 1)$, we reach the formula that allows the regressive computation of all the others $\theta_{n}(\tau)$,

$$
\begin{aligned}
\theta_{n}(\tau)= & {\left[\frac{(n+1)}{\tau}-A\right] \cdot \frac{\theta_{n+1}(\tau)}{(\rho+\Phi)}+\left[\frac{(n+1) \cdot A}{\tau}-B-\frac{(n+1) \cdot \gamma^{2}}{(n+3)}\right] \cdot \frac{\theta_{n+2}(\tau)}{(\rho+\Phi)^{2}}+} \\
& +\left[\frac{(n+1) \cdot B}{\tau}-\frac{(n+1) \cdot A \cdot \gamma^{2}}{(n+3)}\right] \cdot \frac{\theta_{n+3}(\tau)}{(\rho+\Phi)^{3}}-\frac{(n+1) \cdot B \cdot \gamma^{2}}{(n+3)} \cdot \frac{\theta_{n+4}(\tau)}{(\rho+\Phi)^{4}}
\end{aligned}
$$

The last two regressive recurrences, $(108(0))$ and $(108(n))$ can be clustered in only one, which reads like,

General regressive recurrence for functions $\theta_{\mathrm{n}}(\tau)$ (biofilm early stages formation and growth $\underline{\text { model }}$

$$
\begin{aligned}
\theta_{n}(\tau)=\left[\frac{(n+1)}{\tau}-\right. & A] \cdot \frac{\theta_{n+1}(\tau)}{D_{1}}+\left[\frac{(n+1) \cdot A}{\tau}-B-\frac{(n+1) \cdot C}{(n+3)}\right] \cdot \frac{\theta_{n+2}(\tau)}{D_{2}}+ \\
& +\left[\frac{(n+1) \cdot B}{\tau}-\frac{(n+1) \cdot A \cdot C}{(n+3)}\right] \cdot \frac{\theta_{n+3}(\tau)}{D_{3}}-\frac{(n+1) \cdot B \cdot C}{(n+3)} \cdot \frac{\theta_{n+4}(\tau)}{D_{4}}
\end{aligned}
$$

Where:

$$
\begin{aligned}
& A=(1-2 \cdot \Phi-\delta) \\
& B=\Phi \cdot(\Phi+\delta-1) \\
& C=(\rho+\Phi) \cdot(1-\Phi-\delta) \\
& D_{k}=\left\{\begin{array}{l}
\rho \cdot(\rho+\Phi)^{k-1} \ldots \text { for }(n=0) \\
(\rho+\Phi)^{k} \quad \ldots \text { for }(n \geq 1)
\end{array}\right\} \quad \ldots k \text { in the range: } \quad(1 \leq k \leq 4)
\end{aligned}
$$

Remark: The designation "General" for this recurrence means that it is valid in the three cases analysed in the Appendixes A, B, and C, in Part I: 
Case 1: Continuous Biofilm $\quad[\delta<(1-\Phi)]$

Case 2: Patchy Biofilm $\quad[\delta>(1-\Phi)]$

Case 3: Frontier between Continuous and Patchy Biofilm $[\delta=(1-\Phi)]$

Our goal is to apply correctly alike ideas to those applied by Miller [22] but now with this five terms recurrence (FTR) instead of the already known three term recurrence (TTR) classical algorithm.

For start, formula $(108(n))$ is applied at an order $(n+4)$ high enough to allow us to set $\theta_{n+4}(\tau)=0, \theta_{n+3}(\tau)=0$ and $\theta_{n+2}(\tau)=0$. That's to say: these orders, $(n+4),(n+3)$ and $(n+2)$, must be so high that the corresponding fractions of solid support area $\theta_{n+4}(\tau), \theta_{n+3}(\tau)$ and $\theta_{n+2}(\tau)$ represent, in their turn, an accumulation of pilled layers so big that was not yet achieved at any point in the biofilm, at the value of dimensionless time $(\tau)$ under consideration.

In the same formula, $(108(n))$, a value of 1 must be assigned to $\theta_{n+1}(\tau)$ if we follow strictly Miller's criterion (Miller [22]).

However we introduce in this stage an improvement consisting in assign to $\theta_{n+1}(\tau)$ a small positive value, $\varepsilon_{n+1}$, but not necessarily equal to 1 , and preferably less than 1 .

This allows a more conservative increase in absolute values of the successive $\theta_{n-k}(\tau)$ under computation avoiding to reach the upper absolute value limit of Excel, which is $10^{+308}$, or, in inverse reasoning, allows start the recurrence in higher orders $(n+4),(n+3)$ and $(n+2)$ accomplishing this way computations much more extensive in their number of recurrent steps.

So, the first recurrent computation consists in doing $\theta_{n+4}(\tau)=0, \theta_{n+3}(\tau)=0, \theta_{n+2}(\tau)=0$ and $\theta_{n+1}(\tau)=\varepsilon_{n+1}$ where $\left(0<\varepsilon_{n+1} \leq 1\right)$, insert these values in $(108(n))$, and so get the non normalized value of $\theta_{n}(\tau)$, which we designate by $\varepsilon_{n}$.

The second recurrent computation consists in apply once again $(108(n))$, but diminishing 1 to all orders $(n \pm k)$. We designate by $(108(n-1))$ such "new" equation,

$$
\begin{aligned}
\theta_{n-1}(\tau)=\left[\frac{(n)}{\tau}-A\right] & \cdot \frac{\theta_{n}(\tau)}{(\rho+\Phi)}+\left[\frac{(n) \cdot A}{\tau}-B-\frac{(n) \cdot \gamma^{2}}{(n+2)}\right] \cdot \frac{\theta_{n+1}(\tau)}{(\rho+\Phi)^{2}}+ \\
& +\left[\frac{(n) \cdot B}{\tau}-\frac{(n) \cdot A \cdot \gamma^{2}}{(n+2)}\right] \cdot \frac{\theta_{n+2}(\tau)}{(\rho+\Phi)^{3}}-\frac{(n) \cdot B \cdot \gamma^{2}}{(n+2)} \cdot \frac{\theta_{n+3}(\tau)}{(\rho+\Phi)^{4}}
\end{aligned}
$$

Now we insert the former values of $\theta_{n+3}(\tau)=0, \theta_{n+2}(\tau)=0, \theta_{n+1}(\tau)=\varepsilon_{n+1}$ and $\theta_{n}(\tau)=\varepsilon_{n}$ and obtain the non normalized value of $\theta_{n-1}(\tau)$, coherently designated by $\varepsilon_{n-1}$.

This procedure is done repeatedly $(n-2)$ more times till the non normalized value of $\theta_{1}(\tau)$, designed $\varepsilon_{1}$, is calculated.

In general, the $k$-th recurrent computation, being $(1 \leq k \leq n)$, is realized with equation $(108(n-$ $k+1)$ ), which reads,

$$
\begin{aligned}
& \theta_{n-k+1}(\tau)= {\left[\frac{(n-k+2)}{\tau}-A\right] \cdot \frac{\theta_{n-k+2}(\tau)}{(\rho+\Phi)}+\left[\frac{(n-k+2) \cdot A}{\tau}-B-\frac{(n-k+2) \cdot \gamma^{2}}{(n-k+4)}\right] \cdot \frac{\theta_{n-k+3}(\tau)}{(\rho+\Phi)^{2}}+} \\
&+\left[\frac{(n-k+2) \cdot B}{\tau}-\frac{(n-k+2) \cdot A \cdot \gamma^{2}}{(n-k+4)}\right] \cdot \frac{\theta_{n-k+4}(\tau)}{(\rho+\Phi)^{3}}-\frac{(n-k+2) \cdot B \cdot \gamma^{2}}{(n-k+4)} \cdot \frac{\theta_{n-k+5}(\tau)}{(\rho+\Phi)^{4}} \\
&(108(n-k+1))
\end{aligned}
$$

The four initial postulated values, $\theta_{n+4}(\tau)=0, \theta_{n+3}(\tau)=0, \theta_{n+2}(\tau)=0$ and $\theta_{n+1}(\tau)=\varepsilon_{n+1}$ are used in the first recurrent computation. Then, from these, the three values $\theta_{n+3}(\tau)=0, \theta_{n+2}(\tau)=0$ and $\theta_{n+1}(\tau)=\varepsilon_{n+1}$, are still necessary in the second recurrent computation. In the same way, the two values $\theta_{n+2}(\tau)=0$ and $\theta_{n+1}(\tau)=\varepsilon_{n+1}$ are needed for the third computation, and also the value $\theta_{n+1}(\tau)=\varepsilon_{n+1}$ still for the fourth one. 
In the fifth and subsequent recurrent computations only calculated values in former computations are needed. None of the initial fourth postulated values figures anymore.

In all this regressive and recurrent computations, equations $(108(n)),(108(n-1)),(108(n-2))$, $\ldots,(108(n-k+1)), \ldots,(108(3)),(108(2))$ and $(108(1))$ are applied and the non normalized values of $\theta_{n}(\tau), \theta_{n-1}(\tau), \theta_{n-2}(\tau), \ldots, \theta_{n-k+1}(\tau), \ldots, \theta_{3}(\tau), \theta_{2}(\tau)$ and $\theta_{1}(\tau)$ are respectively obtained.

Lastly, the non normalized value of $\theta_{0}(\tau)$, is computed applying equation (108(0)) inserting in it the non normalized values of $\theta_{1}(\tau), \theta_{1}(\tau), \theta_{1}(\tau)$ and $\theta_{1}(\tau)$ obtained, respectively, in the former $n$-th, $(n-1)$-th, $(n-2)$-th and $(n-3)$-th recurrent computations.

In the following Table 1 all the sequence of these recurrent computations for the non normalized values of all the functions $\theta_{k}(\tau)$, being $(0 \leq k \leq n+4)$, is succinctly and clearly explained.

Till this stage we only have the non normalized values of all functions $\theta_{k}(\tau)$. Opportunely we now recall that they relative proportions are already correct.

In the sequel we must add up all those non normalized values and divide all of them by the value of that summation, which, in this way, works like a normalization factor, because the summation of all the fractions $\theta_{k}(\tau)$ must be equal to 1 .

The initial postulated order, from where the recurrent computations must begin to be applied, ought to be large enough for the positive, non normalized, value assigned to $\theta_{n+1}(\tau)$ be much lower or, at least, alike to the reached, non normalized value of $\theta_{0}(\tau)$, at the other extreme of the entire computation. This last scenario is only valid if $\theta_{0}(\tau)$ attained already very low values as consequence of having elapsed enough time since the beginning at $(\tau=0)$ and, therefore, almost support area is just at hand to be completely covered.

In other words: the highest order $(n+1)$ among all the $\theta_{k}(\tau)$ computed and, so, considered not null must be high enough to realty represent a $\theta_{k}(\tau)$ with a positive value, but very low, very near to zero. Only this way the postulated null values of $\theta_{n+2}(\tau), \theta_{n+3}(\tau), \theta_{n+4}(\tau)$, and beyond that can be considered correctly valued.

The aforesaid need for start the recurrent computations in an order $(n+4)$ that can be very high directs us to the new problem of avoid that the maximum value of the non normalized functions $\theta_{k}(\tau)$ don't have an absolute value so high that exceeds the positive numerical limit of Excel spreadsheet. This limit has already been mentioned and is around $10^{+308}$. From our numerical experiments we concluded that this big upper positive limit conjugated with the possibility of start with a postulated value for $\theta_{n+1}(\tau)$ very low, albeit still positive, $\left(\varepsilon_{n+1} \gtrsim 0\right)$, leads always, meaning this for all values of dimensionless time $(\tau)$, to a possible complete and accurate computation of all the functions $\theta_{k}(\tau)$.

Logically, as the dimensionless time $(\tau)$ increases, more and more fractions $\theta_{k}(\tau)$, where order $(k)$ is higher, must be calculated because they represent already absolute positive values not anymore negligible.

Consequently once fixed the model parameters values, in a numerical experiment, as time $(\tau)$ increases a successive need for start the algorithm in an order $(n+4)$ higher, and higher again, come into play. Facing this circumstance the accurate recurrent computation of the kinetic evolution, over a broad range of time $(\tau \geq 0)$, will always imply the construction of a series of similar Generalized Miller's like algorithms in the same spreadsheet, but where the starting computation order $(n+4)$ increases from one to the next algorithm. Bigger values of time $(\tau)$ 
demand bigger initial $(n+4)$ orders as the natural reflex of the physical growth of the biofilm with the continuous accumulation of biomass and concomitant attaining of fractions at solid support area covered by more and more pilled layers.

Table 1

\begin{tabular}{|c|c|c|c|c|c|}
\hline $\begin{array}{l}\text { Computation } \mathrm{n}^{\mathrm{o}} / \\
\text { /Applied equation }\end{array}$ & & Not norma & ed input valu & & \multirow{2}{*}{$\begin{array}{l}\text { Result } \\
\boldsymbol{\theta}_{n}(\tau)\end{array}$} \\
\hline \multirow{2}{*}{$\begin{array}{c}1 \\
(108(n))\end{array}$} & $\boldsymbol{\theta}_{n+4}(\tau)$ & $\boldsymbol{\theta}_{n+3}(\tau)$ & $\boldsymbol{\theta}_{n+2}(\tau)$ & $\theta_{n+1}(\tau)$ & \\
\hline & 0 & 0 & 0 & $0<\varepsilon_{n+1} \leq 1$ & $\varepsilon_{n}$ \\
\hline \multirow{2}{*}{$\begin{array}{c}2 \\
(108(n-1))^{(*)}\end{array}$} & $\boldsymbol{\theta}_{n+3}(\tau)$ & $\boldsymbol{\theta}_{n+2}(\tau)$ & $\theta_{n+1}(\tau)$ & $\boldsymbol{\theta}_{n}(\boldsymbol{\tau})$ & $\theta_{n-1}(\tau)$ \\
\hline & 0 & 0 & $0<\varepsilon_{n+1} \leq 1$ & $\varepsilon_{n}$ & $\varepsilon_{n-1}$ \\
\hline \multirow{2}{*}{$\begin{array}{c}3 \\
(108(n-2))\end{array}$} & $\boldsymbol{\theta}_{n+2}(\tau)$ & $\theta_{n+1}(\tau)$ & $\boldsymbol{\theta}_{n}(\boldsymbol{\tau})$ & $\theta_{n-1}(\tau)$ & $\boldsymbol{\theta}_{n-2}(\tau)$ \\
\hline & 0 & $0<\varepsilon_{n+1} \leq 1$ & $\varepsilon_{n}$ & $\varepsilon_{n-1}$ & $\varepsilon_{n-2}$ \\
\hline \multirow[b]{2}{*}{............. } & ............. & ............. & .............. & .............. & .............. \\
\hline & ............. & ............. & ............. & ............ & ............. \\
\hline \multirow{2}{*}{$\begin{array}{c}\boldsymbol{k}(*) \\
(108(n-k+1))\end{array}$} & $\theta_{n-\mathrm{k}+5}(\tau)$ & $\theta_{n-\mathrm{k}+4}(\tau)$ & $\boldsymbol{\theta}_{n-\mathrm{k}+3}(\boldsymbol{\tau})$ & $\boldsymbol{\theta}_{n-\mathrm{k}+2}(\boldsymbol{\tau})$ & $\theta_{n-\mathrm{k}+1}(\tau)$ \\
\hline & $\varepsilon_{n-k+5}$ & $\varepsilon_{n-k+4}$ & $\varepsilon_{n-k+3}$ & $\varepsilon_{n-k+2}$ & $\varepsilon_{n-k+1}$ \\
\hline \multirow[b]{2}{*}{............ } & ............. & ............. & ............ & ............. & ............. \\
\hline & ............. & .............. & .............. & .............. & ............. \\
\hline \multirow{2}{*}{$\begin{array}{r}n-2 \quad(*) \\
(108(3))\end{array}$} & $\boldsymbol{\theta}_{7}(\boldsymbol{\tau})$ & $\theta_{6}(\tau)$ & $\boldsymbol{\theta}_{5}(\tau)$ & $\boldsymbol{\theta}_{4}(\tau)$ & $\boldsymbol{\theta}_{3}(\tau)$ \\
\hline & $\varepsilon_{7}$ & $\varepsilon_{6}$ & $\varepsilon_{5}$ & $\varepsilon_{4}$ & $\varepsilon_{3}$ \\
\hline
\end{tabular}




\begin{tabular}{|c|c|c|c|c|c|}
\hline \multirow{2}{*}{$\begin{array}{r}n-1 \quad(*) \\
(108(2))\end{array}$} & $\theta_{6}(\tau)$ & $\theta_{5}(\tau)$ & $\boldsymbol{\theta}_{4}(\tau)$ & $\boldsymbol{\theta}_{3}(\boldsymbol{\tau})$ & $\boldsymbol{\theta}_{2}(\boldsymbol{\tau})$ \\
\hline & $\varepsilon_{6}$ & $\varepsilon_{5}$ & $\varepsilon_{4}$ & $\varepsilon_{3}$ & $\varepsilon_{2}$ \\
\hline \multirow{2}{*}{$\begin{array}{c}n \\
(108(1))\end{array}$} & $\boldsymbol{\theta}_{5}(\tau)$ & $\theta_{4}(\tau)$ & $\boldsymbol{\theta}_{3}(\tau)$ & $\boldsymbol{\theta}_{2}(\boldsymbol{\tau})$ & $\theta_{1}(\tau)$ \\
\hline & $\varepsilon_{5}$ & $\varepsilon_{4}$ & $\varepsilon_{3}$ & $\varepsilon_{2}$ & $\varepsilon_{1}$ \\
\hline \multirow{2}{*}{$\begin{array}{r}n+1 \quad(* *) \\
(108(0))\end{array}$} & $\boldsymbol{\theta}_{4}(\boldsymbol{\tau})$ & $\boldsymbol{\theta}_{3}(\tau)$ & $\boldsymbol{\theta}_{2}(\tau)$ & $\theta_{1}(\tau)$ & $\boldsymbol{\theta}_{0}(\tau)$ \\
\hline & $\varepsilon_{4}$ & $\varepsilon_{3}$ & $\varepsilon_{2}$ & $\varepsilon_{1}$ & $\varepsilon_{0}$ \\
\hline \multicolumn{6}{|c|}{$\begin{array}{l}\text { Short legend } \\
\text { (*)-Regressive Recurrences used in the computations numbers }(k) \text {, with }(1 \leq k \leq n) \text { / } \\
\text { / Recurrent equation }(\mathbf{1 0 8}(\boldsymbol{k})) \\
(* *)-\text { Regressive Recurrence used in the computation number }(\boldsymbol{n}+\mathbf{1}) \text { / } \\
\text { /Recurrent equation }(\mathbf{1 0 8}(0))\end{array}$} \\
\hline
\end{tabular}

Appendix A. Continuous biofilm solution

Now follows the solution for continuous biofilm, (case 1).

Lets recall the equation given earlier as eq. (71),

$$
g(s, \tau)=\frac{e^{-(\rho+\Phi) \cdot(1-\Phi-\delta) \cdot \frac{\tau}{s}}}{s}+\int_{0}^{\tau} f(\sigma) \cdot \frac{e^{-(\rho+\phi) \cdot(1-\phi-\delta) \cdot \frac{(\tau-\sigma)}{s}}}{s} \cdot d \sigma
$$

Applying inverse Laplace transform we get,

$$
\begin{aligned}
& G(z, \tau)=J_{0}(2 \cdot \sqrt{(\rho+\Phi) \cdot(1-\Phi-\delta) \cdot \tau \cdot z)}+\cdot \\
& +\int_{0}^{\tau} J_{0}(2 \cdot \sqrt{(\rho+\Phi) \cdot(1-\Phi-\delta) \cdot(\tau-\sigma) \cdot z}) \cdot f(\sigma) \cdot d \sigma
\end{aligned}
$$

At this point, it is convenient to define other functions, related to Bessel functions, but easier for calculation purposes.

We will need to derive partially relatively to $\tau$ and $z$ when the conditions in generating function formalism, before established, must be satisfied. However such derivations applied directly in Bessel functions are somewhat cumbersome.

Such more adequate functions are,

$$
V_{n}(\sigma, \tau, z)=\left[\frac{(\tau-\sigma)}{(\rho+\Phi) \cdot(1-\Phi-\delta) \cdot z}\right]^{n / 2} \cdot J_{n}(2 \cdot \sqrt{(\rho+\Phi) \cdot(1-\Phi-\delta) \cdot(\tau-\sigma) \cdot z})
$$

Their derivatives are very simple to use, according to the following rules,

$$
\frac{\partial V_{n}(\sigma, \tau, z)}{\partial z}=-(\rho+\Phi) \cdot(1-\Phi-\delta) \cdot V_{n+1}(\sigma, \tau, z)
$$




$$
\frac{\partial V_{n}(\sigma, \tau, z)}{\partial \tau}=V_{n-1}(\sigma, \tau, z)
$$

Demonstration of these rules are long and tedious. So we will pursue simply accepting these rules. Inserting (A2) in (A1),

$$
G(z, \tau)=V_{0}(0, \tau, z)+\int_{0}^{\tau} V_{0}(\sigma, \tau, z) \cdot f(\sigma) \cdot d \sigma
$$

Now this solution must satisfy the early designated condition (58), which has not yet been taken in consideration.

For achieve that goal we must determine function $f(\sigma)$.

Let define $f(\sigma)$ by an infinite power series,

$$
f(\sigma)=\sum_{k=0}^{\infty} c_{k} \cdot \sigma^{k}
$$

$\ldots$ and include the power series in (A5),

$$
G(z, \tau)=V_{0}(0, \tau, z)+\sum_{k=0}^{\infty} c_{k} \cdot\left[\int_{0}^{\tau} V_{0}(\sigma, \tau, z) \cdot \sigma^{k} \cdot d \sigma\right]
$$

The integrals,

$$
\int_{0}^{\tau} V_{0}(\sigma, \tau, z) \cdot \sigma^{k} \cdot d \sigma
$$

... can be calculated introducing the definition of the function $V_{0}(\sigma, \tau, z)$ and applying the identity,

$$
\int_{0}^{\tau}(\tau-\sigma)^{i} \cdot \sigma^{k} \cdot d \sigma=\frac{k ! i !}{(k+i+1) !} \cdot \tau^{k+i+1}
$$

The result, which we here only indicate, is,

$$
\int_{0}^{\tau} V_{0}(\sigma, \tau, z) \cdot \sigma^{k} \cdot d \sigma=k ! \cdot V_{k+1}(0, \tau, z)
$$

The consequent sequel is that (A7) becomes,

$$
G(z, \tau)=V_{0}(0, \tau, z)+\sum_{k=0}^{\infty} c_{k} \cdot k ! \cdot V_{k+1}(0, \tau, z)
$$

Rules (A3) and (A4) came now into play to accomplish already defined condition (58) in this last equation.

Calculating the partial derivatives of the generating function in (A10) they reads,

$$
\begin{gathered}
\frac{\partial G(z, \tau)}{\partial \tau}=V_{-1}(0, \tau, z)+\sum_{k=0}^{\infty} c_{k} \cdot k ! \cdot V_{k}(0, \tau, z) \\
\frac{\partial G(z, \tau)}{\partial z}=-(\rho+\Phi) \cdot(1-\Phi-\delta) \cdot\left[V_{1}(0, \tau, z)+\sum_{k=0}^{\infty} c_{k} \cdot k ! \cdot V_{k+2}(0, \tau, z)\right]
\end{gathered}
$$

Easily one can prove, 


$$
V_{-n}(\sigma, \tau, z)=\left[\frac{(-1)(\rho+\Phi)(1-\Phi-\delta)}{(\tau-\sigma)}\right]^{n} \cdot V_{n}(\sigma, \tau, z)
$$

Formula (A13) avoids the utilization of functions of negative order.

It can be used straightaway in (A11),

$$
\frac{\partial G(z, \tau)}{\partial \tau}=-\frac{(\rho+\Phi) \cdot(1-\Phi-\delta) \cdot z}{\tau} \cdot V_{1}(0, \tau, z)+\sum_{k=0}^{\infty} c_{k} \cdot k ! \cdot V_{k}(0, \tau, z)
$$

At last the two partial derivatives of the generating function at $z=\tau$ can be written,

$$
\begin{aligned}
& {\left[\frac{\partial G(z, \tau)}{\partial \tau}\right]_{z=\tau}=-(\rho+\Phi) \cdot(1-\Phi-\delta) \cdot V_{1}(0, \tau, \tau)+\sum_{k=0}^{\infty} c_{k} \cdot k ! \cdot V_{k}(0, \tau, \tau)} \\
& {\left[\frac{\partial G(z, \tau)}{\partial z}\right]_{z=\tau}^{\infty}=-(\rho+\Phi) \cdot(1-\Phi-\delta) \cdot\left[V_{1}(0, \tau, \tau)+\sum_{k=0}^{\infty} c_{k} \cdot k ! \cdot V_{k+2}(0, \tau, \tau)\right]}
\end{aligned}
$$

The left hand side of early condition (58) is (A15), and is now possible to write it putting the functions $V_{k}(0, \tau, \tau)$ in increasing order $n$,

$\left[\frac{\partial G(z, \tau)}{\partial \tau}\right]_{z=\tau}=c_{0} \cdot V_{0}(0, \tau, \tau)+\left[c_{1}-(\rho+\Phi) \cdot(1-\Phi-\delta)\right] \cdot V_{1}(0, \tau, \tau)+\sum_{k=2}^{\infty} c_{k} \cdot k ! \cdot V_{k}(0, \tau, \tau)$

In the same way, the right hand side of (58) can be rewritten substituting there (A10) and (A16). And also with the functions $V_{k}(0, \tau, \tau)$ in increasing order $n$,

$$
\begin{aligned}
(2 \cdot \Phi+\delta-1) \cdot G(\tau, \tau)-\frac{\Phi}{(\rho+\Phi)} \cdot\left(\frac{\partial G}{\partial z}\right)_{z=\tau}= \\
=(2 \cdot \Phi+\delta-1) \cdot V_{0}(0, \tau, \tau)+\left[(2 \cdot \Phi+\delta-1) \cdot c_{0}-\Phi \cdot(1-\Phi-\delta)\right] \cdot V_{1}(0, \tau, \tau)+ \\
\quad+\sum_{k=0}^{\infty}\left[\Phi \cdot(1-\Phi-\delta) \cdot c_{k} \cdot k !+(2 \cdot \Phi+\delta-1) \cdot c_{k+1} \cdot(k+1) !\right] \cdot V_{k+2}(0, \tau, \tau)
\end{aligned}
$$

Early obtained equation (58) implies to equalize the right hand sides of (A17) and (A18). Such equalization only will be valid if the factors affecting each function $V_{k}(0, \tau, \tau)$ are equal on those both right hand sides. In this way a system of algebraic equations is established and, from that system, all the coefficients $c_{k}$ defined without ambiguity. And, at last, the generating function is completely defined.

The aforesaid system, extracted from (A17) and (A18), is,

$c_{0}=(2 \cdot \Phi+\delta-1)$

$c_{1}-(\rho+\Phi) \cdot(1-\Phi-\delta)=\Phi \cdot(1-\Phi-\delta)+(2 \cdot \Phi+\delta-1) \cdot c_{0}$

$c_{2} \cdot 2 !=\Phi \cdot(1-\Phi-\delta) \cdot c_{0}+(2 \cdot \Phi+\delta-1) \cdot c_{1}$

$c_{3} \cdot 3 !=\Phi \cdot(1-\Phi-\delta) \cdot c_{1}+(2 \cdot \Phi+\delta-1) \cdot c_{2} \cdot 2$ !

$c_{4} \cdot 4 !=\Phi \cdot(1-\Phi-\delta) \cdot c_{2} \cdot 2 !+(2 \cdot \Phi+\delta-1) \cdot c_{3} \cdot 3 !$

$c_{k+2} \cdot(k+2) !=\Phi \cdot(1-\Phi-\delta) \cdot c_{k} \cdot k !+(2 \cdot \Phi+\delta-1) \cdot c_{k+1} \cdot(k+1) !$

$(A 19(k+2))$

The two first (A19 (0)) and (A19 (1)) are preliminary conditions and all the others have the general form given by $(\mathrm{A} 19(\mathrm{k}+2))$.

This last one is a difference equation which form can be also put like,

$$
b_{k+2}+A \cdot b_{k+1}+B \cdot b_{k}=0
$$

... where, 


$$
\begin{gathered}
b_{k}=c_{k} \cdot k ! \\
A=(1-2 \cdot \Phi-\delta) \\
B=\Phi \cdot(\Phi+\delta-1)
\end{gathered}
$$

The general solution of (A20) is known elsewhere,

$$
b_{k}=\frac{\left[b_{1}+b_{0}\left(A+r_{1}\right)\right]}{\left(r_{1}-r_{2}\right)} \cdot r_{1}^{k}-\frac{\left[b_{1}+b_{0}\left(A+r_{2}\right)\right]}{\left(r_{1}-r_{2}\right)} \cdot r_{2}^{k}
$$

... where the roots are,

$$
r_{1}=\frac{-A+\sqrt{A^{2}-4 \cdot B}}{2} \quad r_{2}=\frac{-A-\sqrt{A^{2}-4 \cdot B}}{2}
$$

In the case under analysis, inserting (A22) and (A23) in (A25),

$$
r_{1}=\Phi \quad r_{2}=(\Phi+\delta-1)
$$

Substitution in (A24) and consideration of (A21) leads to the coefficients $c_{k}$,

$$
c_{k} \cdot k !=\frac{1}{(1-\delta)} \cdot\left\{\left[c_{1}+c_{0} \cdot(1-\Phi-\delta)\right] \cdot \Phi^{k}-\left(c_{1}-c_{0} \cdot \Phi\right) \cdot(\Phi+\delta-1)^{k}\right\}
$$

The coefficient $c_{0}$ is given by (A19 (0)) and $c_{1}$ can be obtained by (A19 (1)),

$$
c_{1}=(1-\Phi-\delta) \cdot(\rho+2 \cdot \Phi)+(2 \cdot \Phi+\delta-1)^{2}
$$

Now $c_{0}$ and $c_{1}$ are eliminated from (A27) by substitution,

$$
c_{k}=\frac{1}{(1-\delta) \cdot k !} \cdot\left\{[\rho \cdot(1-\Phi-\delta)+\Phi \cdot(1-\delta)] \cdot \Phi^{k}+(\rho+1-\delta) \cdot(\Phi+\delta-1)^{k+1}\right\}
$$

Formula (A29) is valid for $k \geq 1$.

For $k=0$, the formula (A19 (0)) must be used for calculate $c_{0}$.

Considering (A21) we can use, from now on, the coefficients $b_{k}$ or the $c_{k}$.

For sake of economy in notation is better to chose, from now on, only the coefficients $b_{k}$.

What is achieved till now about these coefficients can be appropriately summarized,

$$
b_{k}=\left\{\begin{array}{l}
(2 \cdot \Phi+\delta-1) \ldots \text { for } k=0 \\
\frac{\left\{[\rho \cdot(1-\Phi-\delta)+\Phi \cdot(1-\delta)] \cdot \Phi^{k}+(\rho+1-\delta) \cdot(\Phi+\delta-1)^{k+1}\right\}}{(1-\delta)}
\end{array}\right.
$$

$$
\text { ... for } k \geq 1
$$

Taking in consideration (A21) and substituting these two expressions in (A10) one gets the final wanted formula for the generating function,

$$
\begin{aligned}
G(z, \tau) & =V_{0}(0, \tau, z)+(2 \cdot \Phi+\delta-1) \cdot V_{1}(0, \tau, z)+ \\
& +\frac{1}{(1-\delta)} \cdot \sum_{k=1}^{\infty}\left\{[\rho \cdot(1-\Phi-\delta)+\Phi \cdot(1-\delta)] \cdot \Phi^{k}+(\rho+1-\delta) \cdot(\Phi+\delta-1)^{k+1}\right\} \cdot V_{k+1}(0, \tau, z)
\end{aligned}
$$

Functions $Q_{n}(\tau)$ are defined by the early eq. (60). But let first generalize (A3) for $n$ derivations (is convenient change $n$ by $k$ in (A3) and then to derive $n$ times),

$$
\frac{\partial^{n} V_{k}(\sigma, \tau, z)}{\partial z^{n}}=(-1)^{n} \cdot[(\rho+\Phi) \cdot(1-\Phi-\delta)]^{n} \cdot V_{k+n}(\sigma, \tau, z)
$$

Applying in (A31), 


$$
\frac{\partial^{n} G(z, \tau)}{\partial z^{n}}=(-1)^{n} \cdot[(\rho+\Phi) \cdot(1-\Phi-\delta)]^{n} \cdot\left\{V_{n}(0, \tau, z)+b_{0} \cdot V_{n+1}(0, \tau, z)+\sum_{k=1}^{\infty} b_{k} \cdot V_{n+k+1}(0, \tau, z)\right\}
$$

Where $b_{0}$ and $b_{k}$ are defined by $(A 30(0))$ and $(A 30(k))$.

Lastly, functions $Q_{n}(\tau)$, for $n \geq 1$,

$$
Q_{n}(\tau)=-\frac{(-1)^{n} \cdot \rho}{(\rho+\Phi) \cdot(1-\Phi-\delta)^{n}} \cdot\left(\frac{\partial^{n} G}{\partial z^{n}}\right)_{z=\tau} \ldots \ldots \text { for }(n \geq 1)
$$

Resulting, for $n \geq 1$,

$$
Q_{n}(\tau)=\rho \cdot(\rho+\Phi)^{n-1} \cdot\left\{V_{n}(0, \tau, \tau)+b_{0} \cdot V_{n+1}(0, \tau, \tau)+\sum_{k=1}^{\infty} b_{k} \cdot V_{n+k+1}(0, \tau, \tau)\right\}
$$

The fractions $\theta_{n}(\tau)$, for $n \geq 1$ are reached with the substitution, already named (28),

$$
\theta_{n}(\tau)=e^{-(\rho+2 \cdot \Phi-\delta-1) \cdot \tau} \cdot Q_{n}(\tau)
$$

The fractions $\theta_{0}(\tau)$ is obtained from (A30), putting $z=\tau$, because $Q_{0}(\tau)=G(\tau, \tau)$, and then also applying (28).

The desired solution for case 1 , in terms of Bessel functions of first kind, $J_{n}(x)$, is eq. (80) which can now be found in section 5.2 .

For such achievement one only need to apply the early defined formula (81), but inverting, so making explicit the functions $J_{n}(2 \cdot \sqrt{(\rho+\Phi) \cdot(1-\Phi-\delta) \cdot(\tau-\sigma) \cdot z})$,

$$
J_{n}(2 \cdot \sqrt{(\rho+\Phi) \cdot(1-\Phi-\delta) \cdot(\tau-\sigma) \cdot z})=\left[\frac{(\rho+\Phi) \cdot(1-\Phi-\delta) \cdot z}{(\tau-\sigma)}\right]^{n / 2} \cdot V_{n}(\sigma, \tau, z)
$$

Appendix B. Patchy biofilm solution

Let recall that the solution (117) is only the solution for the before named case 1, corresponding to continuous biofilm.

As signalled in the preceding box, (117) corresponds to the condition $\delta<(1-\Phi)$.

The "complementary" condition, $\delta>(1-\Phi)$, designated before as case 2, relates the evolution towards a steady state where the bare fraction $\theta_{0}$ don't never get null. In physical terms this means the formation, at times large enough, of a patchy biofilm.

The solution for case 2 can be obtained simply profiting solution (117), being aware that whenever root $\sqrt{1-\Phi-\delta}$ occurs substitution by $i \cdot \sqrt{\Phi+\delta-1}$ must be done.

Consequently, in (117) the variable $x$ and the coefficients $\alpha_{n}$ and $\beta_{k}$ are modified as follows, Variable $x$ changes this way,

$$
\begin{gathered}
x=2 \cdot \tau \cdot \sqrt{(\rho+\Phi) \cdot(1-\Phi-\delta)} \\
2 \cdot \tau \cdot \sqrt{(\rho+\Phi) \cdot(1-\Phi-\delta)}=i \cdot 2 \cdot \tau \cdot \sqrt{(\rho+\Phi) \cdot(\Phi+\delta-1)} \\
i \cdot 2 \cdot \tau \cdot \sqrt{(\rho+\Phi) \cdot(\Phi+\delta-1)}=i \cdot x^{*}
\end{gathered}
$$

So the change in variable $x$ reads,

$$
x \rightarrow\left(i \cdot x^{*}\right)
$$

Coefficient $\alpha_{n}$ changes this way,

$$
\begin{gathered}
\frac{\rho \cdot(\rho+\Phi)^{n-1}}{\sqrt{(\rho+\Phi)^{n} \cdot(1-\Phi-\delta)^{n}}}=\frac{\rho \cdot(\rho+\Phi)^{n-1}}{i^{n} \cdot \sqrt{(\rho+\Phi)^{n} \cdot(\Phi+\delta-1)^{n}}} \\
\frac{\rho \cdot(\rho+\Phi)^{n-1}}{i^{n} \cdot \sqrt{(\rho+\Phi)^{n} \cdot(\Phi+\delta-1)^{n}}}=i^{-n} \cdot a_{n}^{*}
\end{gathered}
$$

So the change in coefficient $\alpha_{n}$ reads, 


$$
\alpha_{n} \rightarrow\left(i^{-n} \cdot \alpha_{n}^{*}\right)
$$

And the new coefficient $\alpha_{n}^{*}$ is now, for all values of $n$,

$$
\alpha_{n}^{*}=\left\{\begin{array}{l}
1 \ldots \text { for } n=0 \\
\frac{\rho \cdot(\rho+\Phi)^{n-1}}{\sqrt{(\rho+\Phi)^{n} \cdot(\Phi+\delta-1)^{n}}} \ldots \text { only for } n \geq 1
\end{array}\right.
$$

Coefficient $\beta_{1}$ changes this way,

$$
\begin{gathered}
\beta_{1}=\frac{(2 \cdot \Phi+\delta-1)}{\sqrt{(\rho+\Phi) \cdot(1-\Phi-\delta)}} \\
\frac{(2 \cdot \Phi+\delta-1)}{\sqrt{(\rho+\Phi) \cdot(1-\Phi-\delta)}}=\frac{(2 \cdot \Phi+\delta-1)}{i \cdot \sqrt{(\rho+\Phi) \cdot(\Phi+\delta-1)}} \\
\frac{(2 \cdot \Phi+\delta-1)}{i \cdot \sqrt{(\rho+\Phi) \cdot(\Phi+\delta-1)}}=i^{-1} \cdot \beta_{1}^{*}
\end{gathered}
$$

So the change in coefficient $\beta_{1}$ reads,

$$
\beta_{1} \rightarrow\left(i^{-1} \cdot \beta_{1}^{*}\right)
$$

Coefficient $\beta_{k}$, for $(k \geq 2)$, changes this way,

$$
\beta_{k}=\frac{\left\{[\rho \cdot(1-\Phi-\delta)+\Phi \cdot(1-\delta)] \cdot \Phi^{k-1}+(\rho+1-\delta) \cdot(\Phi+\delta-1)^{k}\right\}}{(1-\delta) \cdot \sqrt{(\rho+\Phi)^{k} \cdot(1-\Phi-\delta)^{k}}}
$$

... but the numerator can be written using the $b_{k}$ definition,

$$
b_{k-1}=\left\{[\rho \cdot(1-\Phi-\delta)+\Phi \cdot(1-\delta)] \cdot \Phi^{k-1}+(\rho+1-\delta) \cdot(\Phi+\delta-1)^{k}\right\}
$$

(observe that in the numerator $b_{k-1}$ doesn't exist any square root, so remains the same) ... thus lightning the notation,

$$
\begin{gathered}
\beta_{k}=\frac{b_{k-1}}{\sqrt{(\rho+\Phi)^{k} \cdot(1-\Phi-\delta)^{k}}} \\
\frac{b_{k-1}}{\sqrt{(\rho+\Phi)^{k} \cdot(1-\Phi-\delta)^{k}}}=\frac{b_{k-1}}{i^{k} \cdot \sqrt{(\rho+\Phi)^{k} \cdot(\Phi+\delta-1)^{k}}} \\
\frac{b_{k-1}}{i^{k} \cdot \sqrt{(\rho+\Phi)^{k} \cdot(\Phi+\delta-1)^{k}}}=i^{-k} \cdot \beta_{k}^{*}
\end{gathered}
$$

So the change in coefficient $\beta_{k}$, wherever occurs, reads,

$$
\beta_{k} \rightarrow\left(i^{-k} \cdot \beta_{k}^{*}\right)
$$

And the new coefficient $\beta_{k}^{*}$ is now, for all values of $n$,

$\beta_{0}^{*}=1$

$\beta_{1}^{*}=\frac{(2 \cdot \Phi+\delta-1)}{\sqrt{(\rho+\Phi) \cdot(\Phi+\delta-1)}}$

$\beta_{k}^{*}=\frac{\left\{[\rho \cdot(1-\Phi-\delta)+\Phi \cdot(1-\delta)] \cdot \Phi^{k-1}+(\rho+1-\delta) \cdot(\Phi+\delta-1)^{k}\right\}}{(1-\delta) \cdot \sqrt{(\rho+\Phi)^{k} \cdot(\Phi+\delta-1)^{k}}}$ ... only for $k \geq 2$

Lastly and easily the solution for case 2 (patchy biofilm) can now be written taking the early deduced in Appendix A, eq.(80) as starting point, and applying the preceding obtained changes in coefficients.

Step by step: 


$$
\theta_{n}(\tau)=\alpha_{n} \cdot e^{y} \cdot \sum_{k=0}^{\infty} \beta_{k} \cdot J_{n+k}(x)
$$

Using (B1), (B2) and (B5),

$$
\alpha_{n} \cdot e^{y} \cdot \sum_{k=0}^{\infty} \beta_{k} \cdot J_{n+k}(x)=i^{-n} \cdot a_{n}^{*} \cdot e^{y} \cdot \sum_{k=0}^{\infty} i^{-k} \cdot \beta_{k}^{*} \cdot J_{n+k}\left(i \cdot x^{*}\right)
$$

Recalling that $J_{n}(i \cdot x)=i^{n} \cdot I_{n}(x)$,

$$
i^{-n} \cdot a_{n}^{*} \cdot e^{y} \cdot \sum_{k=0}^{\infty} i^{-k} \cdot \beta_{k}^{*} \cdot J_{n+k}\left(i \cdot x^{*}\right)=i^{-n} \cdot a_{n}^{*} \cdot e^{y} \cdot \sum_{k=0}^{\infty} i^{-k} \cdot \beta_{k}^{*} \cdot i^{n+k} \cdot I_{n+k}\left(x^{*}\right)
$$

Associating all the factors $i^{-n}, i^{-k}$ and $i^{n+k}$,

$$
i^{-n} \cdot a_{n}^{*} \cdot e^{y} \cdot \sum_{k=0}^{\infty} i^{-k} \cdot \beta_{k}^{*} \cdot i^{n+k} \cdot I_{n+k}\left(x^{*}\right)=a_{n}^{*} \cdot e^{y} \cdot \sum_{k=0}^{\infty} \beta_{k}^{*} \cdot I_{n+k}\left(x^{*}\right)
$$

Finally, concatenating the already obtained eq. (80) with (B7), then with (B8) and lastly with (B9) the searched solution reads,

$$
\theta_{n}(\tau)=a_{n}^{*} \cdot e^{y} \cdot \sum_{k=0}^{\infty} \beta_{k}^{*} \cdot I_{n+k}\left(x^{*}\right)
$$

This is eq. (81) and represents the solution for case 2 (Patchy biofilm). Can now be found completely written down in section 5.2.

\section{Appendix C. Frontier between continuous and patchy biofilm solution}

The solution for case 3 can be easily deduced by induction after doing the corresponding simplifications in the initial system of differential-difference master equations, just before any other step.

It is so simple that we only indicate the solution,

$\theta_{0}(\tau)=e^{-\rho}$

$(C 1(0))$

$\theta_{n}(\tau)=e^{-(\rho+\Phi) \cdot \tau} \cdot \frac{\rho \cdot(\rho+\Phi)^{n-1}}{\Phi^{n}}\left\{e^{\Phi \tau}-\sum_{k=0}^{n-1} \frac{(\tau \cdot \Phi)^{k}}{k !}\right\} \quad \ldots$ for $(n>0)$

This solution is coherent with earlier found solution (80) for case 1 and it is important and interesting to check that coherence. We can achieve that goal written solution eq. (80) in this alternative form,

$\underline{\text { Solution for case 1: continuous biofilm }[\delta<(1-\Phi)] \text { (alternative representation) }}$

$$
\theta_{n}(\tau)=A_{n} \cdot e^{y} \cdot \sum_{k=0}^{\infty} B_{k} \cdot \frac{J_{n+k}(x)}{\gamma^{n+k}}
$$

Where:

$$
\begin{aligned}
& x=2 \cdot \tau \cdot \gamma \\
& y=(1-\rho-2 \cdot \Phi-\delta) \cdot \tau \\
& \gamma=\sqrt{(\rho+\Phi) \cdot(1-\Phi-\delta)} \\
& A_{0}=1 \\
& A_{n}=\rho \cdot(\rho+\Phi)^{n-1} \ldots \text { only for } n \geq 1 \\
& B_{0}=1 \\
& B_{1}=(2 \cdot \Phi+\delta-1)
\end{aligned}
$$




$$
B_{k}=\frac{\left\{[\rho \cdot(1-\Phi-\delta)+\Phi \cdot(1-\delta)] \cdot \Phi^{k-1}+(\rho+1-\delta) \cdot(\Phi+\delta-1)^{k}\right\}}{(1-\delta)} \quad \ldots \text { only for } k \geq 2
$$

When $\delta=1-\Phi, \gamma=0$ and, in the summation in (C2), each Bessel function term assumes the following value,

$$
\begin{gathered}
\frac{J_{n+k}(x)}{\gamma^{n+k}}=\frac{1}{\gamma^{n+k}} \cdot \sum_{i=0}^{\infty} \frac{(-1)^{i} \cdot(\tau \cdot \gamma)^{n+k+2 \cdot i}}{i ! \cdot(n+k+i) !} \\
\frac{1}{\gamma^{n+k}} \cdot \sum_{i=0}^{\infty} \frac{(-1)^{i} \cdot(\tau \cdot \gamma)^{n+k+2 \cdot i}}{i ! \cdot(n+k+i) !}=\sum_{i=0}^{\infty} \frac{(-1)^{i} \cdot \tau^{n+k+2 \cdot i} \cdot \gamma^{2 \cdot i}}{i ! \cdot(n+k+i) !}
\end{gathered}
$$

Consequently, if $\gamma=0$,

$$
\frac{J_{n+k}(x)}{\gamma^{n+k}}=\frac{\tau^{n+k}}{(n+k) !}
$$

And inserting in (C2),

$$
\theta_{n}(\tau)=A_{n} \cdot e^{y} \cdot \sum_{k=0}^{\infty} B_{k} \cdot \frac{\tau^{n+k}}{(n+k) !}
$$

Coefficients $B_{k}$ simplify,

$$
B_{k}=\Phi^{k} \ldots \text { for }(k \geq 0)
$$

... as well as $y$,

$$
y=-(\rho+\Phi) \cdot \tau
$$

Now we conclude that,

$$
\theta_{0}(\tau)=e^{-(\rho+\Phi) \cdot \tau} \cdot \sum_{k=0}^{\infty} \Phi^{k} \cdot \frac{\tau^{k}}{k !}
$$

... and,

... and,

$$
e^{-(\rho+\Phi) \cdot \tau} \cdot \sum_{k=0}^{\infty} \Phi^{k} \cdot \frac{\tau^{k}}{k !}=e^{-(\rho+\Phi) \cdot \tau} \cdot e^{\Phi \cdot \tau}
$$

... and also,

$$
e^{-(\rho+\Phi) \cdot \tau} \cdot e^{\Phi \cdot \tau}=e^{-\rho \cdot \tau}
$$

Concatenating those last three equalities one obtains $(C 1(0))$,

And, when $(n \geq 1)$,

$$
\theta_{n}(\tau)=\rho \cdot(\rho+\Phi)^{n-1} \cdot e^{-(\rho+\Phi) \cdot \tau} \cdot \sum_{k=0}^{\infty} \Phi^{k} \cdot \frac{\tau^{n+k}}{(n+k) !}
$$

$$
\rho \cdot(\rho+\Phi)^{n-1} \cdot e^{-(\rho+\Phi) \cdot \tau} \cdot \sum_{k=0}^{\infty} \Phi^{k} \cdot \frac{\tau^{n+k}}{(n+k) !}=e^{-(\rho+\Phi) \cdot \tau} \cdot \frac{\rho \cdot(\rho+\Phi)^{n-1}}{\Phi^{n}} \cdot \sum_{k=0}^{\infty} \frac{(\Phi \cdot \tau)^{n+k}}{(n+k) !}
$$

But, once the following identity, holds,

$$
\sum_{k=0}^{\infty} \frac{(\Phi \cdot \tau)^{n+k}}{(n+k) !}=e^{-\rho \cdot \tau}-\sum_{k=0}^{n-1} \frac{(\Phi \cdot \tau)^{k}}{k !}
$$

... we get,

$$
\theta_{n}(\tau)=e^{-(\rho+\Phi) \cdot \tau} \cdot \frac{\rho \cdot(\rho+\Phi)^{n-1}}{\Phi^{n}}\left\{e^{\Phi \tau}-\sum_{k=0}^{n-1} \frac{(\tau \cdot \Phi)^{k}}{k !}\right\} \ldots \text { for }(n>0)
$$

... which is equal to $(C 1(n))$, 
Acknowledgments. The authors are grateful to Professors Luis Sousa Lobo and Manuel Esquível for their opportune and fruitful discussions on this topic.

\section{$\underline{\text { References }}$}

1. White J. A., Schmidt, J. W., and Bennett G. K., Analysis of Queueing Systems, Academic Press, New York (1975)

2. Kleinrock, L., Queueing Systems, vol.1: Theory, Wiley, New York (1975)

3. Akimaru,H., and Kawashima K., Teletraffic: Theory and Applications ( $2^{\circ}$ ed), Springer, London (1999)

4. Yan, Houmin, Yin, George, Zhang, Qing, Eds., Stochastic Processes, Optimization, and Control Theory: Applications in Financial Engineering, Queueing Networks, and Manufacturing Systems, , Springer (2006)

5. Abate, J., and Whitt W., Calculating Time-Dependent Performance Measures for the M/M/1 Queue. IEEE Transactions on Communications, vol. 37, No. 10, (1989), pp. 1102-1104

6. C.G. Cassandras and S. Lafortune, Introduction to Discrete Event Systems ( $2^{\circ}$ edition), Springer, (2010)

7. Marvin K. Simon, Mohamed-Slim Alouini, Digital Communication over Fading Channels, 2nd Edition, Wiley-IEEE Press, (2005)

8. Thomas G. Robertazzi, Computer Networks and Systems: Queueyng Theory and Performance Evaluation, (3rd edition), Springer (2000)

9. Ryszard Syski, Introduction to congestion theory in telephone systems, $2^{a}$ edition, North-Holland, (1986)

10. Goodrich, F. C., Nucleation Rates and the Kinetics of Particle Growth. II. The Birth and Death Process, Proc.Roy.Soc.Lond. A 277 (1964) 167-182

11. Carvalho, J. M., From discrete biofilm model formation to queuing transient analysis, https://run.unl.pt/handle/10362/77252, Ph. D. thesis (Chemical and Biochemical Engineering), Nova University of Lisbon, Lisbon (2018)

12. Amparo Gil, Javier Segura and Nico M. Temme, Numerical Methods for Special Functions, SIAM, (2007)

13. H. C. Thacher, "New Backward Recurrences for Bessel Functions”, Math. Comp., vol. 33, no 146, 744-764, (1979)

14. Clarke A. B., A Waiting Line Process of Markov Type, The Annals of Mathematical Statistics, Vol. 27, No. 2 (Jun., 1956), pp. 452-459.

15. Pinney, E., Ordinary Difference-Differential Equations, University of California Press (1958)

16. Bellman, R., and Cooke, K.L., Differential-Difference Equations, Academic Press (1963)

17. El'sgol'ts, L.E. e Norkin,S.B., Introduction to the Theory and Application of Differential Equations with Deviating Arguments, Academic Press. (1973)

18. Clarke, A. B., On time-dependent waiting line processes (Abst.) Ann.Math.Statist. 24, 491-492 (1953).

19. Saaty, T.L., Elements of Queueing Theory with applications, McGraw-Hill (1961)

20. Srivastava, H.M., and Kashyap, B.R.K., Special Functions in Queuing Theory, Academic Press (1982)

21. Abramowitz, M. and Stegun, I., Eds., Handbook of Mathematical Functions. Washington, D.C.: National Bureau of Standards, (1964). Reprinted by Dover Publications, New York, (1968)

22. Miller J.C.P., Bessel Functions, vol.II in Mathematical Tables, Cambridge University Press, London and New York (1952)

23. Zhang S. e Jin J.,Computation of Special Functions Wiley-Interscience (1996) 